\title{
Wittig reagents for chemoselective sulfenic acid ligation enables global site stoichiometry analysis and redox-controlled mitochondrial targeting
}

\section{Yunlong Shi}

Scripps Research Institute

Ling Fu

Beijing Institute of Lifeomics

Jing Yang

State Key Laboratory of Proteomics, Beijing Proteome Research Center, National Center for Protein

Sciences, Beijing Institute of Lifeomics, Beijing 102206 https://orcid.org/0000-0001-8486-273X

Kate Carroll ( $\nabla$ kcarroll@scripps.edu )

Scripps Research Institute https://orcid.org/0000-0002-7624-9617

\section{Article}

Keywords: Triphenylphosphonium ylides, Wittig reagents

Posted Date: November 30th, 2020

DOI: https://doi.org/10.21203/rs.3.rs-110280/v1

License: (c) (i) This work is licensed under a Creative Commons Attribution 4.0 International License.

Read Full License

Version of Record: A version of this preprint was published at Nature Chemistry on September 16th, 2021.

See the published version at https://doi.org/10.1038/s41557-021-00767-2. 
1 Wittig reagents for chemoselective sulfenic acid ligation enables global site

2 stoichiometry analysis and redox-controlled mitochondrial targeting

3

4 Yunlong Shi ${ }^{1}$, Ling Fu ${ }^{2,3}$, Jing Yang ${ }^{2 *}$ and Kate S. Carroll ${ }^{1 *}$

$5 \quad$ 'Department of Chemistry, The Scripps Research Institute, Jupiter, Florida 33458, United States

$6 \quad{ }^{2}$ State Key Laboratory of Proteomics, Beijing Proteome Research Center, National Center for Protein

7 Sciences $•$ Beijing, Beijing Institute of Lifeomics, Beijing 102206, China

$8{ }^{3}$ Innovation Institute of Medical School, Medical College, Qingdao University, Qingdao 266071, China

$9 \quad$ *e-mail: kcarroll@scripps.edu

10 *e-mail: yangiing54@hotmail.com 
12 Triphenylphosphonium ylides, known as Wittig reagents, are one of the most commonly used tools in 13 synthetic chemistry. Despite their considerable versatility, Wittig reagents have not yet been explored 14 for their utility in biological applications. Here, we introduce a new chemoselective ligation reaction that 15 harnesses the reactivity of Wittig reagents and the unique chemical properties of sulfenic acid, a pivotal 16 post-translational cysteine modification in redox biology. The reaction, which generates a covalent bond 17 between the ylide nucleophilic $\alpha$-carbon and electrophilic $\gamma$-sulfur is highly selective, rapid, and affords 18 robust labeling under a range of biocompatible reaction conditions, including in living cells. We highlight 19 the broad utility of this conjugation method to enable site-specific proteome-wide stoichiometry analysis 20 of S-sulfenylation, visualize redox-dependent changes in mitochondrial cysteine oxidation, and redox21 triggered TPP generation for controlled delivery of small molecules to mitochondria. 
24 While modern methods provide access to molecules of increasing complexity, traditional organic 25 transformations remain an essential cornerstone of synthetic chemistry. Their fundamental value is 26 increasingly underscored by their potential to be repurposed as biocompatible reactions, with novel 27 application in living systems ${ }^{1,2}$. Pioneering examples of named reactions that have been broadly 28 employed to discover new biology are the Staudinger reaction ${ }^{3}$ and the Huisgen 1,3-dipolar cycloaddition $^{4}$. Application of conventional organic reactions in biological settings also enables us to revisit and expand our understanding of known reactions, including the development of new substrates and, by extension, novel products.

a

Wittig Reagents: Originally used for alkene synthesis

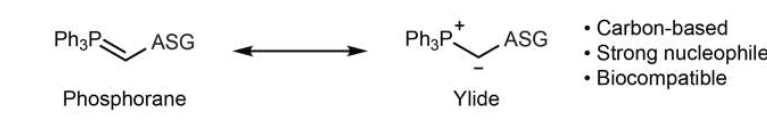

b

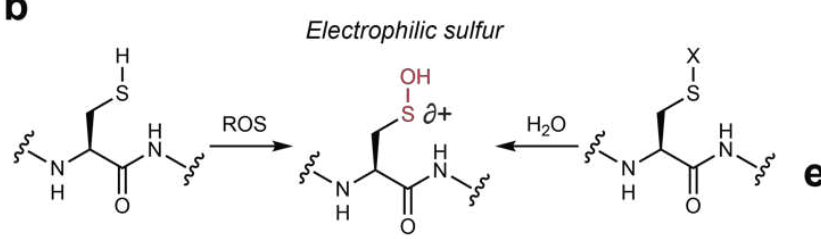

C

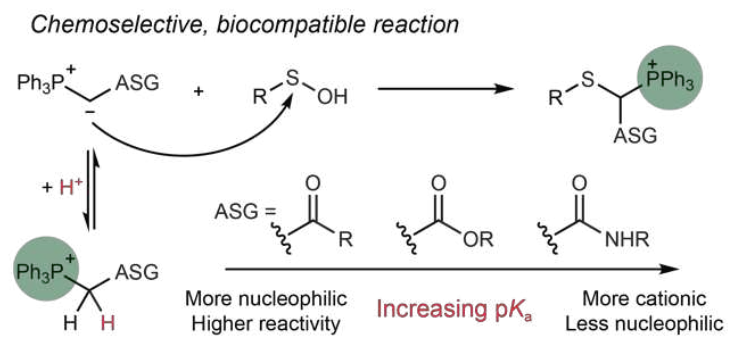

d

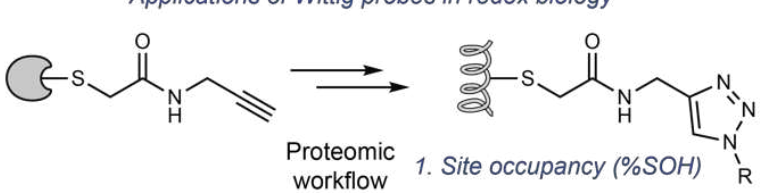

\section{e}<smiles></smiles>

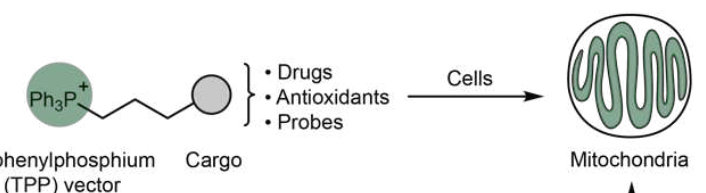

Triphenylphosphium Cargo
(TPP) vector

Mitochondria

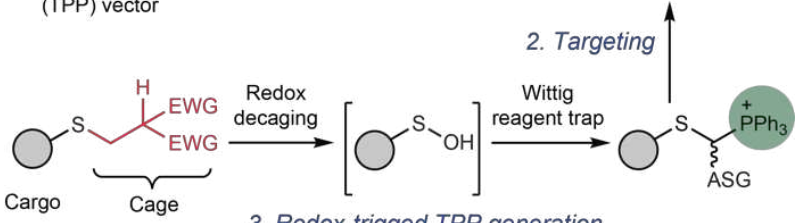

3. Redox-trigged TPP generation

Figure 1 | Repurposing triphenylphosphonium ylides as probes for electrophilic sulfur in proteins. a, Wittig reagents can be illustrated as the phosphorane or ylide form. With anion-stabilizing groups (ASG), Wittig reagents act as water-compatible carbon-based nucleophiles. b, Sulfenic acids are formed via two major pathways: direct oxidation or hydrolysis of polarized sulfur species. c, Biocompatible reaction between sulfenic acids and stabilized Wittig reagents. d, Determination of S-sulfenylation site occupancy. After proteomic workflow, labeled protein thiols (top) and sulfenic acids (bottom) yielded isotopomers. e, Taking advantage of the TPP group installed after reaction with the Wittig reagent enables direct or redox-triggered cargo delivery to mitochondria.

32 The Wittig reaction is a widespread technique used for the preparation of alkenes through the use of a triphenylphosphonium yilde (Wittig reagent) ${ }^{5}$. Ylides are neutral molecules with opposite charges on adjacent atoms. In the context of the Wittig reagent, the triphenylphosphonium (TPP) group holds a delocalized positive charge that stabilizes the nucleophilic carbanion. Although commonly written as the 
zwitterionic ylide form which highlights the strong nucleophilicity of the carbanion (Fig. 1a). Anion stabilizing substituents can further modulate the $\alpha$-carbon, and produce more stable ylides, some of which become compatible in aqueous media 6 . Despite the considerable utility of Wittig reagents in organic synthesis, versatility from the tuning of the ylide $\alpha$ - substituents, and compatibility of the reaction with aqueous systems, phosphonium yildes have not been mined for their use in chemoselective, biocompatible reactions.

In the interest of developing new reactions with application in cells and keeping the nucleophilic character of phosphonium ylides in mind, we hypothesized that the chemically unique electrophilic sulfur in sulfenic acid, a post-translational modification of protein cysteines (Fig. 1b), could serve as the novel reaction partner in vitro and cells. Sulfenic acid is generated through one of two routes: Direct oxidation of a thiolate by reactive oxygen species such as hydrogen peroxide produced during cellular signaling and metabolism or by hydrolysis of sulfenyl halides, cyclic sulfenamides, and very polarized nitrosothiols and disulfides $^{7}$ (Supplementary Fig. 1a). Sulfenic acids have a $p K_{a}$ of 6-7 in proteins, indicating that both protonated and deprotonated states are accessible at physiological $\mathrm{pH}^{8}$. If stabilized by the microenvironment, the thiol-sulfenic acid (or sulfenate) pair can operate as a switch triggered by redox changes, regulating protein function, structure, and localization ${ }^{9}$. Alternatively, the electrophilic sulfur in sulfenic acid may condense with a protein or low-molecular-weight thiol to form a disulfide or, under conditions of excess oxidative stress, may be oxidized further to sulfinic and sulfonic acids. In either scenario - as stabilized or transient intermediate - sulfenic acids are central modifications in the domain of biological redox-regulation ${ }^{10,11}$ (Supplementary Fig. 1b). Furthermore, our group ${ }^{8,12-16}$ and others ${ }^{17,18}$ have shown that the electrophilic character of sulfur in sulfenic acid is chemically distinct from protein electrophiles, including modifications to amino or thiol functional groups, making this species an ideal candidate to examine for reactivity with Wittig reagents.

2 With the unexamined potential of nucleophilic Wittig reagents in biological applications and the advancement of our understanding of electrophilic sulfenic acid modifications in biology and 
64 pharmacology in mind, we embarked upon a new chemical journey. Here, we report the reaction between

65 the ylide nucleophilic $\alpha$-carbon in Wittig reagents and the electrophilic $\gamma$-sulfur of sulfenic acid to generate

66 a C-S bond (Fig. 1C). The reaction is highly selective, rapid, and affords robust labeling under a range of biocompatible reaction conditions, including in living cells. We highlight the broad utility of this conjugation method to enable site-specific proteome-wide stoichiometry analysis of S-sulfenylation, visualize redox-dependent changes in mitochondrial cysteine oxidation, and redox-triggered TPP generation for controlled delivery of small molecules to mitochondria (Fig. 1d,e).

Table 1 | Surveying Wittig reagent reactivity with sulfenic acid.
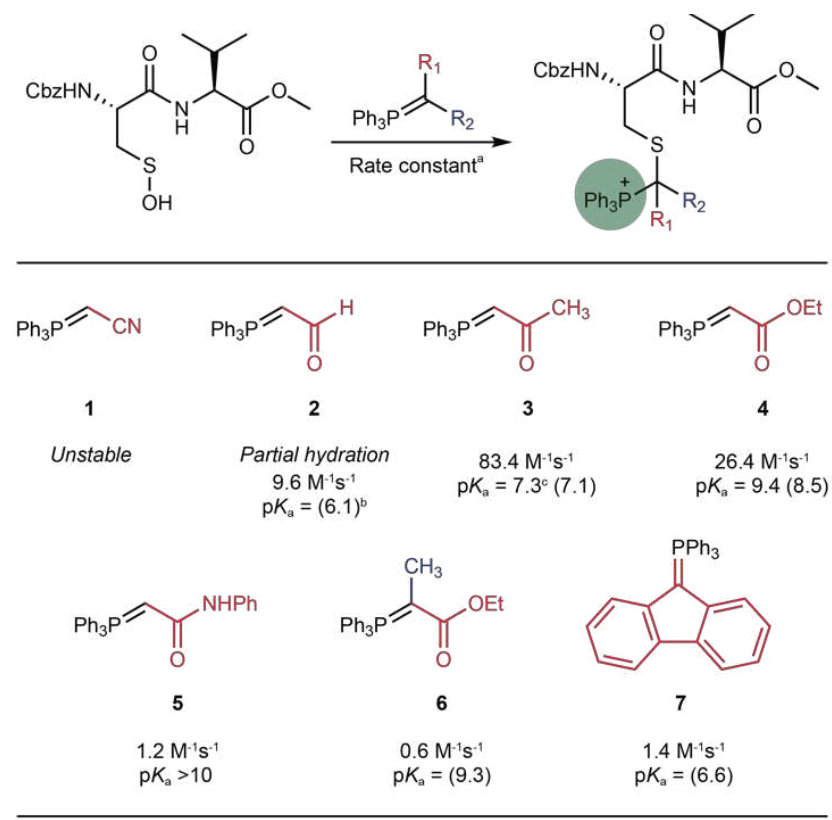

a Rate constants were obtained in $\mathrm{ACN}: \mathrm{NaOAc}(1: 2)$ at $\mathrm{pH}=4.9$.

${ }^{\mathrm{b}}$ Literature $\mathrm{pK} \mathrm{K}_{\mathrm{a}}$ values (in parentheses) were measured in DMSO. ${ }^{39}$

72

${ }^{\circ}$ Experimental $\mathrm{p} K_{\mathrm{a}}$ values were obtained in $\mathrm{ACN}: \mathrm{H}_{2} \mathrm{O}$ as described in Supplementary Figs. 3-4.

\section{Results}

74 Screening Wittig reagent reactivity. We initiated our study with Wittig reagents functionalized with

75 anion stabilizing groups (1-7). A dipeptide cyclic sulfenamide model compound that generates a sulfenic

76 acid in situ when dissolved in water ${ }^{8}$ was used to survey the reactivity of these reagents (Table 1 ). Nitrile-

77 and aldehyde-functionalized Wittig reagents (1-2) underwent hydrolysis in organic-aqueous buffer at $\mathrm{pH}$

787.4 and were not further pursued. Mono-substituted derivatives including ketone $\mathbf{3}$, ester $\mathbf{4}$ or amide $\mathbf{5}$,

79 and di-substituted Wittig reagents 6-7, formed stable products with the sulfenic acid model in quantitative 
80 yield. Compounds $\mathbf{3}$ and $\mathbf{4}$ showed exceptional reactivity, requiring the collection of kinetic data at lower

$81 \mathrm{pH}$ (Supplementary Fig. 2). In general, reaction rates increased with stronger electron withdrawing 82 substituents, but dramatically decreased with an additional $\alpha$-substituent, presumably due to steric 83 hindrance. Kinetic data was also in agreement with the measured $p K_{a}$ of the conjugate acids of 84 compounds 3-5 (Supplementary Figs. 3 and 4), strongly suggesting that the fraction of Wittig reagent in 85 neutral form is the key factor controlling the reaction rates.

Preparation and evaluation of Wittig-alkyne (WYne) reagents in chemical models. With success in sulfenic acid trapping using the model dipeptide with carbonyl-, ester- and amide-substituted Wittig reagents, we synthesized the corresponding derivatives with a "clickable" alkyne handle, termed Wittigalkyne or "WYne" probes (Fig. 2a). WYneC (8) was prepared via alkylation of ketone 3, while esterification or amidation of bromoacetone bromide followed by treatment of triphenylphosphine furnished WYneO (9) and WYneN (10). In the case of amide derivatives 5 and 10, deprotonation by aqueous base led to rapid hydrolysis, because the weakly electron withdrawing amide group was unable to provide sufficient stabilization (Fig. 2b). Consequently, these compounds were prepared and used as protonated salts. All WYne probes (8-10) were stable in dry, powdered form, and compatible with aqueous media under open air during a typical period of analysis, although WYneO was mildly affected by hydrolytic decomposition (Fig. 2a). WYne probes exhibited robust reactivity with the sulfenic acid dipeptide model, analogous to parent Wittig reagents 3-5 (Fig. 2a-d and Supplementary Fig. 5). WYneC exhibited a 10 -fold increase in kinetics compared to our a benzo[c][1,2]thiazine-based sulfenic acid probe, $\mathrm{BTD}^{15}$ and was roughly 1,500 -times faster than $\mathrm{DYn}-2^{13}$, an early reagent based on the $1,3-$ cyclohexanedione scaffold (Fig. 2d, structures of BTD and DYn-2 shown in Supplementary Fig. 1c,d). WYneO was nearly as reactive as WYneC, while WYneN exhibited slower kinetics due to a decrease in nucleophilicity imparted by the amide substituent (Fig. 2a,d). Overall, WYne probes were successfully reacted with the dipeptide sulfenic acid model to give the expected S-adduct with rate constants ranging from 160 to $15,000 \mathrm{M}^{-1} \cdot \mathrm{s}^{-1}$ at physiological $\mathrm{pH}$. 
a

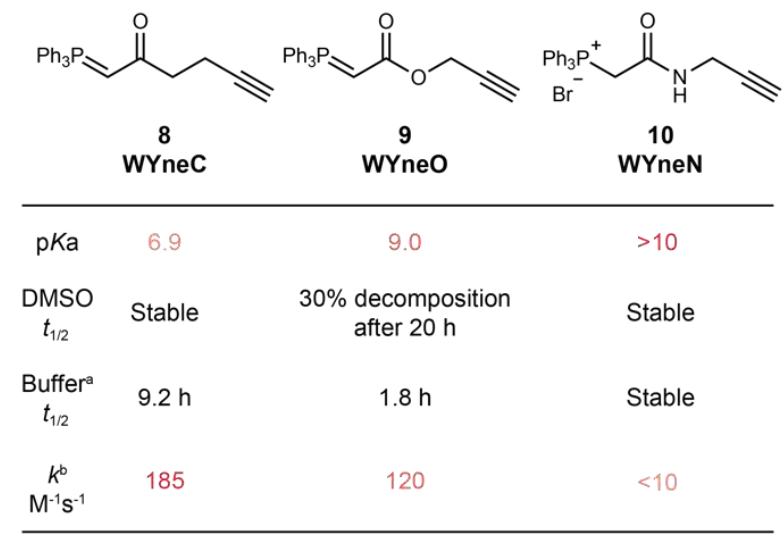

a $50 \mathrm{mM}$ HEPES, $100 \mathrm{mM} \mathrm{NaCl}, \mathrm{pH}=7.4 \quad{ }^{\mathrm{b}} \mathrm{ACN}: \mathrm{NaOAc}(1: 2) \mathrm{pH}=4.9$

b

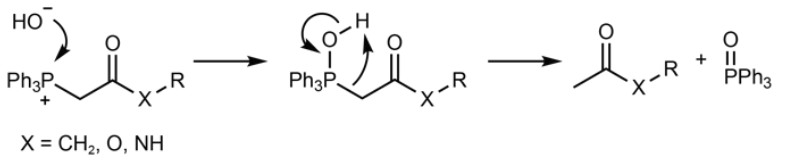

C

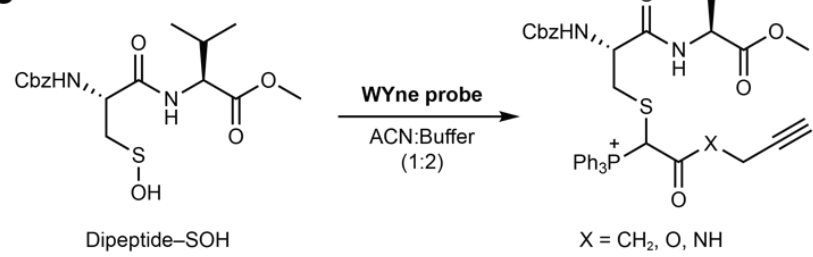

d

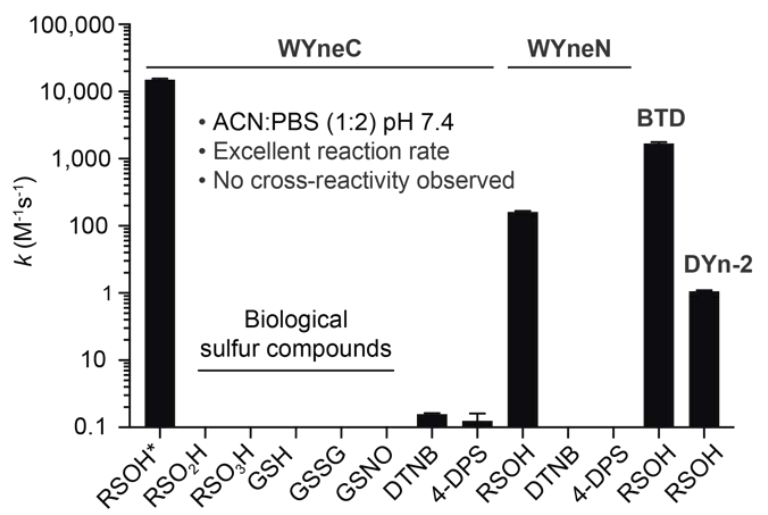

S. cerevisiae Gpx3

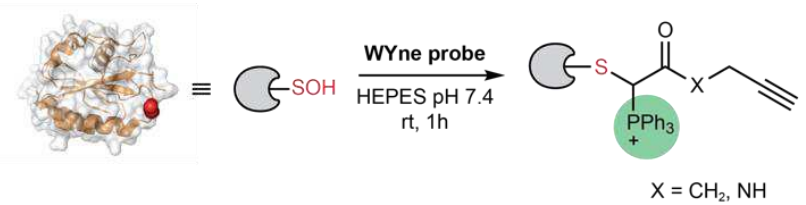
C64,82S Gpx3-SH C64,82S Gpx3-SOH $\quad$ C64,82S Gpx3-SO $\quad$ H $\quad$ C64S Gpx3-SS (36-82)

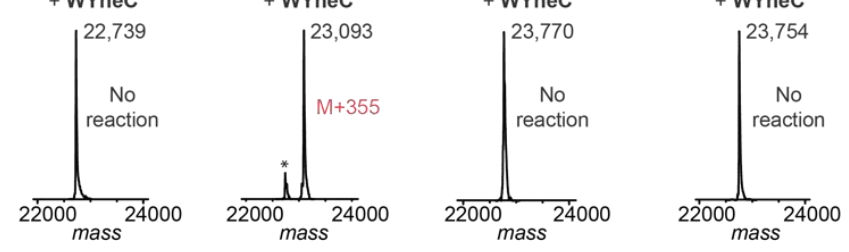

C64,82S Gpx3-SH $\quad$ C64,82S Gpx3-SOH $\quad$ C64,82S Gpx3-SOH $\quad$ C64,82S Gpx3-SO $\mathrm{H}_{2}$ + WYneN + WYneN + WYneN (pH 8.5) + WYneN

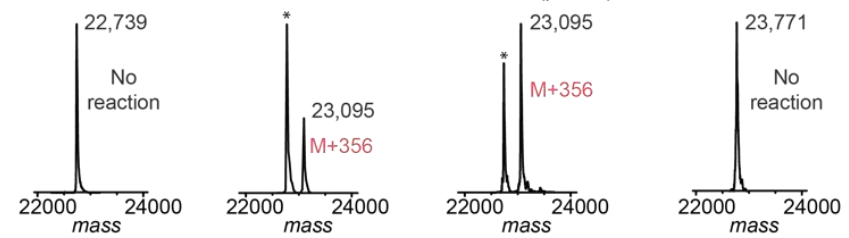

cos
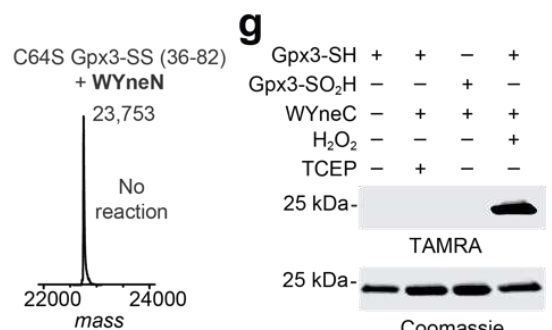

h

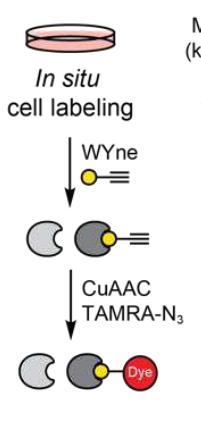

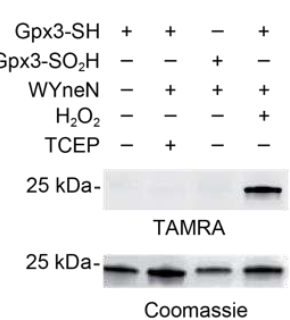

$\mathrm{Gp} \times 3-\mathrm{SH}++-+$

WYneN -+++

$\mathrm{H}_{2} \mathrm{O}_{2}---+$

TCEP - + - -

Coomassie

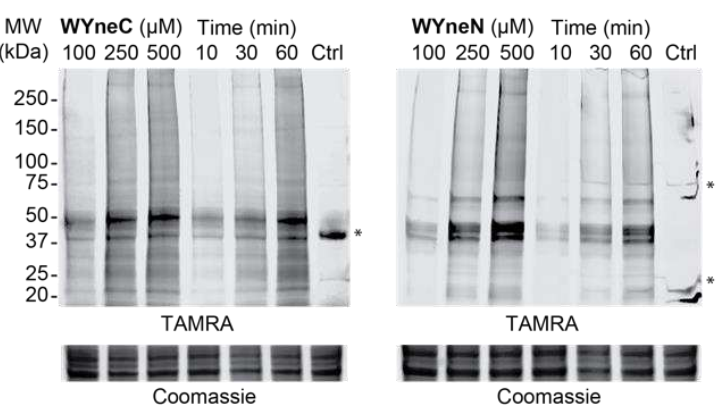

Figure 2 | WYne probe reactivity with sulfenic acid in complex biological settings. a, Physical property and kinetic profile of the WYne probes. b, Base-promoted hydrolysis facilitated cleavage of the TPP group from WYne probes. c, A small molecule sulfenic acid model (dipeptide-SOH) was used to kinetically evaluate WYne probes. d, Reaction of WYne probes and various sulfur species in pH 7.4 aqueousorganic buffer. Owing to rapid kinetics, the reaction between WYneC and dipeptide-SOH (R-SOH) was performed at pH 4.9 and the rate constant extrapolated to $\mathrm{pH} 7.4$ (see Supplementary Methods). Reactivity with other biological sulfur species was not observed. e, Glutathione peroxidase 3 (Gpx3, from S. cerevisiae) as a model to study protein sulfenic acids. f, Intact protein MS analyses suggested that WYne probes exclusively targeted the sulfenic acid form of Gpx3 and not the thiol, sulfinic acid or disulfide forms. $\mathbf{g}$, In-gel fluorescence detection of Gpx3 sulfenic acid. h, In situ labeling of A549 cells with WYne probes showed time and dose-dependence.

107 Next, we interrogated the selectivity of WYne probes 8-10 against related redox cysteine modifications.

108 WYne probes did not exhibit cross-reactivity with glutathione, glutathione disulfide, S-nitrosoglutathione, 
a slight, but measurable reaction, with chemically activated disulfides such as Ellman's reagent (DTNB)

111 or 4,4'-dipyridyl disulfide (4-DPS) (Fig. 2d and Supplementary Fig. 7). Although these polarized disulfides

112 are not naturally occurring in proteins and reacted with WYneC at significantly decreased reaction rates

113 ( 100,000-fold) as compared to sulfenic acid. Various aldehydes including 4-nitrobenzaldehyde,

114 pyridoxal, isovaleraldehyde, and formaldehyde were also surveyed for potential cross-reactions. WYne

115 probes 8-10 reacted stoichiometrically with sulfenic acid, without interference from electrophilic

116 aldehydes (Supplementary Fig. 8). Lastly, we evaluated the stability of the S-adduct formed between

117 WYne probes 8-10 and the dipeptide (Fig. 2c and Supplementary Fig. 9). WYne S-adducts were 118 generally stable in neutral and acidic buffers, but the WYneN S-adduct was sensitive to basic conditions $119(\mathrm{pH}>10)$ resulting in a loss of the TPP group. S-adduct of WYneC was stable under millimolar 120 concentration of reducing agent, while WYneO and WYneN adducts were labile (Supplementary Fig. 9);

121 therefore, strong reducing environments should be avoided when using these two probes. Collectively, 122 these data indicate that WYne probes react rapidly and selectively with sulfenic acids under aqueous 123 conditions at neutral $\mathrm{pH}$, boding well for the utility of these reagents in more complex systems.

Profiling WYne reactivity in more complex biological settings. Bolstered by our success in chemical models, we moved on to more targets with greater biological relevance and complexity. To this end, we examined WYne probe reactivity in C64,82S glutathione peroxidase 3 (Gpx3; Fig. 2e). This Gpx3 variant possesses one redox-sensitive cysteine, C36 that can be readily oxidized to sulfenic acid using stoichiometric amounts of hydrogen peroxide $\left(\mathrm{H}_{2} \mathrm{O}_{2}\right)$ and has been well-validated as a model for the study of protein sulfenic acid reactivity ${ }^{8,14,19}$. Intact mass spectrometry (MS) analysis demonstrated that Gpx3 C36 sulfenic acid (Gpx3-SOH) formed the anticipated adduct with WYne probes in high yield, while reduced Gpx3 (Gpx3-SH), Gpx3 C36 sulfinic acid $\left(\mathrm{Gpx3}-\mathrm{SO}_{2} \mathrm{H}\right)$ or a $\mathrm{Gpx3}$ variant with an intramolecular disulfide bond (Gpx3 C64S-SS) were not modified (Fig. 2f). Having verified protein-WYne adducts by MS, we conjugated the alkyne tags to TAMRA-azide using the copper(I)-catalyzed alkyne-azide cycloaddition (CuAAC, "click" reaction) and visualized the resulting products by in-gel fluorescence (Fig. 2g). Signal was detected in WYne-treated Gpx3-SOH but not in $\mathrm{Gpx3}-\mathrm{SH}$ or $\mathrm{Gpx3}-\mathrm{SO}_{2} \mathrm{H}$, consistent with 
137 the findings in our intact MS analysis. Finally, we assessed the ability of WYneC and WYneN probes to 138 enter live cells and directly label endogenous targeted proteins (Fig. 2h). Protein labeling was time and dose-dependent; moreover, the high efficiency of WYne probes afforded the opportunity to reduce the concentration applied to cells ( $\geq 10$-fold) compared to that traditionally required for analysis with BTD, DYn-2 and earlier reagents $(\leq 500 \mu \mathrm{M}$ versus $5 \mathrm{mM})$ with minimal cytotoxic effect (Supplementary Fig. 10). Together, these feasibility studies indicate that the reaction between functionalized Wittig reagents and the electrophilic sulfur in sulfenic acid represents a viable strategy to label proteins in the test tube and cells.

Examining WYne probe reactivity and selectivity through chemoproteomics. Encouraged by robust reactivity and selectivity in sulfenic acid dipeptide and protein models, alongside cell permeability and lack of cytotoxic effects, we moved forward with a more rigorous evaluation of WYne probes in our wellestablished chemoproteomics platform ${ }^{13,20,21}$ for site-specific mapping of the S-sulfenylome (Fig. 3a). In brief, native lysates prepared from A549 cells were labeled in separate reactions with WYne probes 810. After trypsin digestion, the resulting probe-labeled peptides were conjugated to light or heavy azidoUV-cleavable-biotin reagents (1:1) via CuAAC. Light and heavy biotinylated peptides were mixed equally, captured on streptavidin beads, and photoreleased for MS-shotgun proteomics for identification and quantification. Probe-labeled peptides covalently conjugated to light or heavy tags yield an isotopic signature in which only peptide assignments with a light/heavy ratio close to 1.0 are recognized as true identifications. Surprisingly, our initial database search targeting intact modifications derived from WYne probes yielded only 474, 147, and 0 probe-labeled sites for WYneC, WYneO, and WYneN, respectively

(Fig. 3b,c and Supplementary Table 1), whereas many unassigned MS1 peaks with the isotopic signature $\left(R_{\mathrm{L} / \mathrm{H}} \approx 1.0\right)$ were identified in the raw data. Hence, we conducted a blind search as previously described ${ }^{22}$, which revealed that all three WYne probes underwent a loss of the TPP moiety, likely caused by base161 promoted hydrolysis during tryptic digestion (Fig. 3d). The cleaved products exhibited different patterns 162 of MS/MS fragmentation compared to the corresponding intact modification derived (Supplementary Fig. 163 11) and the intensity of the former was dramatically higher than the latter (Fig. 3b). Fortuitously, WYneN- 
a

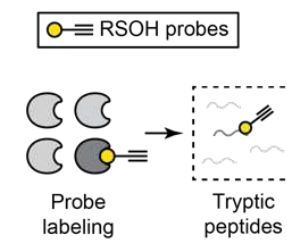

b

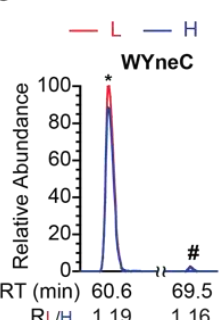

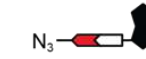
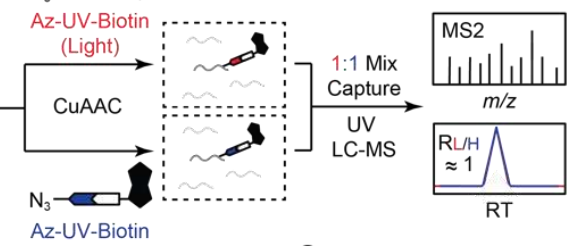

Az-UV-Biotin

(Heavy)

* Cleaved mod.
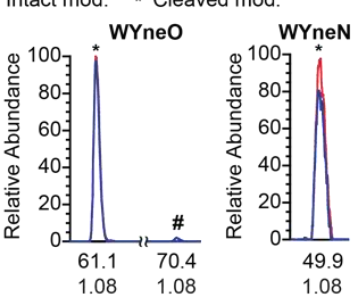

C

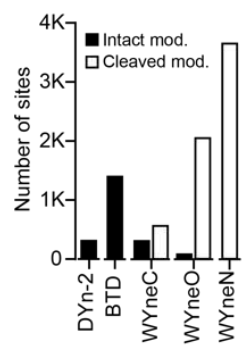

d
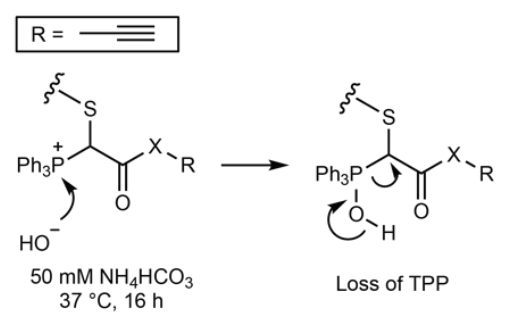

Loss of TPP

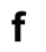

e Insitu WYneN f
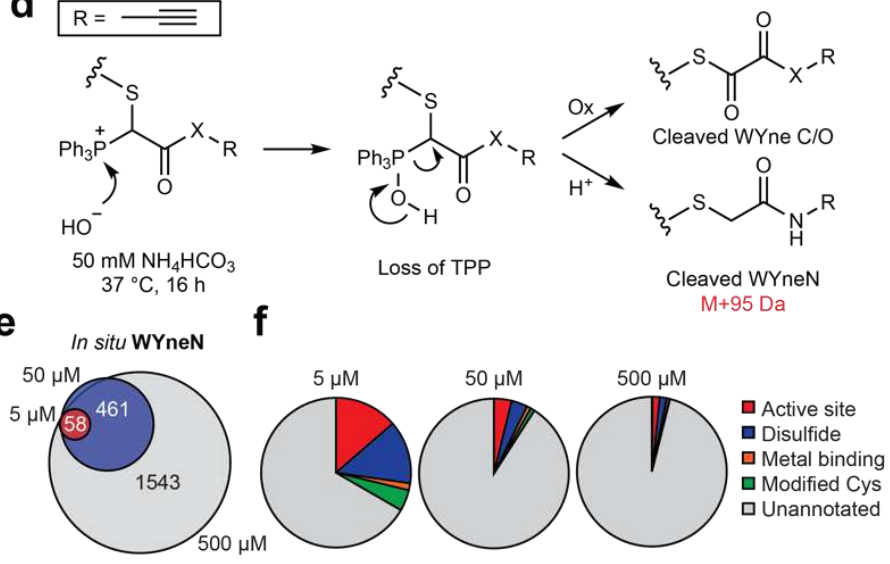

Cleaved WYneN $\mathrm{M}+95 \mathrm{Da}$ g

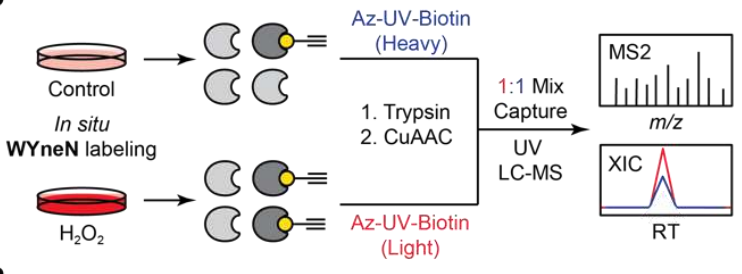

h

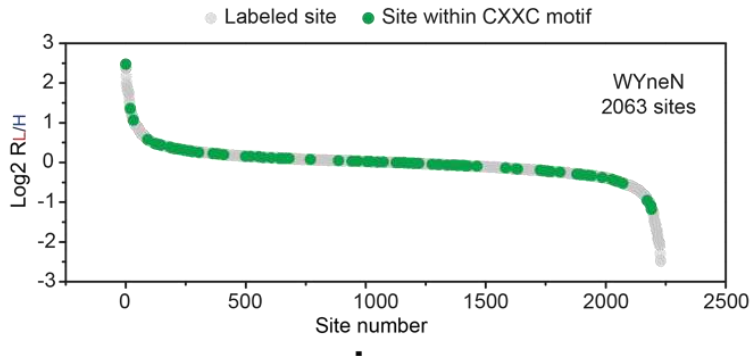

i

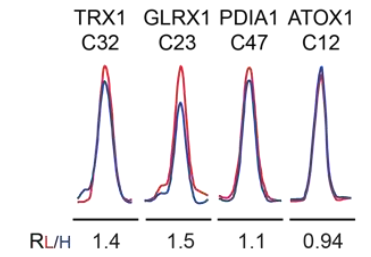

j $\mathrm{H}_{2} \mathrm{O}_{2}+{ }_{-}^{\mathrm{TRX} 1}+\mathrm{H}_{2} \mathrm{O}_{2}^{\mathrm{TRX}}+{ }_{-}^{\mathrm{C}} 32 \mathrm{~A}$ $10 \mathrm{kDa}--10 \mathrm{kDa}$ TAMARA TAMARA $10 \mathrm{kDa}-\underset{\text { Coomassie }}{-} 10 \mathrm{kDa}-\frac{\mathrm{Coomassie}}{-}$ $\begin{array}{cc}10 \mathrm{kDa}-\frac{\text { GLRX1 }}{\text { TAMARA }} & 10 \mathrm{kDa}-\frac{\text { ATOX1 }}{\text { TAMARA }} \\ 10 \mathrm{kDa}-\frac{{ }_{\text {Coomassie }}}{2} 10 \mathrm{kDa}-\frac{\overbrace{\text { Coomassie }}}{}\end{array}$

k $\begin{array}{lllll}\text { TRX1-SH } & \text { TRX1 TRX1 C32A } & \text { TRX1-SS } & \text { GLRX1 ATOX1 }\end{array}$ $+\mathrm{H}_{2} \mathrm{O}_{2}+\mathrm{H}_{2} \mathrm{O}_{2}$

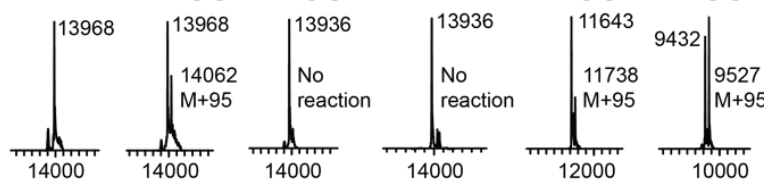

Figure 3 | Profiling S-sulfenylation dynamics with WYneN in cells. a, Quantitative proteomics provides unequivocal identifications of SOH probe-labeling sites in the A549 proteome. b, Extracted ion chromatograms (XICs) of the isotopically labeled peptide (VPTANVSVVDLTCR derived from GAPDH) adducts derived from WYne probes. The intact (\#) and TPP-cleaved (*) adducts derived from each probe are shown in the same scale. c, Bar chart showing the number of sulfenylated sites profiled by using different $\mathrm{SOH}$-specific probes. d, Plausible mechanism for the formation of TPP-cleaved peptide adducts derived from WYne probes. e, Venn diagram showing the overlap of sulfenylated sites mapped by using WYneN with different doses for in situ labeling. f, Pie charts showing the percentage of functionally annotated cysteine sites from different WYneN-dosing groups. g, Quantitative chemoproteomic profiling of dynamic S-sulfenylation changes in $\mathrm{A}_{549}$ cells upon $\mathrm{H}_{2} \mathrm{O}_{2}$ treatment. $\mathbf{h}$, Rank order of the determined $\mathrm{R}_{\mathrm{LH}}$ values of WYneN-labeled sites from A549 cells treated with or without $\mathrm{H}_{2} \mathrm{O}_{2}$. Those sites within redox-sensitive CXXC motifs were annotated in green color. $\mathbf{i}$, Representative XICs showing for the changes in WYneN-labeled cysteines from TRX1, GLRX1, PDIA1 and ATOX1, with the profiles for light and heavy-labeled peptides in red and blue, respectively. $\mathbf{j}$, Validation of CXXC protein labeling by in-gel fluorescence. $\mathbf{k}$, Intact protein MS shows that TPP-cleavage occurs during the labeling of CXXC proteins.

derived cysteine modifications were quantitatively transformed into the TPP-cleaved products, providing

focused subsequent proteomic validation studies on WYneN. 
$37^{\circ} \mathrm{C}, 2 \mathrm{~h}$ ), WYneN enabled the identification of 2,063 S-sulfenylated sites in A549 cells growing under steady-state redox conditions (i.e., no addition of exogenous oxidant) drastically outperforming BTD, which yielded only 126 sites (Fig. 3e and Supplementary Table 2). Remarkably, when applied at only 5 $\mu \mathrm{M}, 59$ S-sulfenylated sites could still be mapped onto 54 proteins using WYneN (Fig. $3 f$ and Supplementary Table 2). Although a dose-dependent increase in identified sulfenic acid sites was not unexpected, we observed that lower concentrations of WYneN correlated with a higher percentage of functionally important cysteine residues (e.g., active site, disulfide, metal binding) mapped across sites (Fig. 3f). This finding suggests that high WYneN reactivity exhibited by individual sulfenic acid modifications positively associates with functional importance. We also investigated the selectivity of WYneN on a proteomic scale using the Open-pFind algorithm to re-analyze the in situ dataset of WYneN labeling by a targeted search of all polar amino acids ${ }^{23}$. WYneN predominately labeled cysteine residues at both a site- (90.0\%, Supplementary Fig. 12a) and spectral-level (99.8\%, Supplementary Fig. 12b). Furthermore, WYneN did not perturb the level of reduced protein cysteines in cells and no observable or statistically significant changes in thiol-labeling could be identified (Supplementary Fig. 12c-e). In particular, the reduced form of several hyperreactive redox sites, such as GAPDH C152, PRDX6 C47, and GSTO1 C32, showed little response to the WYneN probe (Supplementary Fig. 12f). These quantitative global proteomic data, together with rigorous studies in small molecules and recombinant protein, demonstrate that WYneN selectively ligates sulfenic acid with high selectivity and rapid kinetics under a variety of biocompatible conditions.

Profiling S-sulfenylation dynamics with WYneN in cells. With validated WYneN probe in hand, we next applied this chemical tool to ratiometrically quantify dynamic changes in S-sulfenylation in situ after perturbation with $\mathrm{H}_{2} \mathrm{O}_{2}$. Specifically, $\mathrm{A} 549$ cells were treated with vehicle or with $\mathrm{H}_{2} \mathrm{O}_{2}$, and then treated with WYneN (Fig. 3g). Probe-tagged proteomes with and without oxidant treatment were digested into tryptic peptides, conjugated with light and heavy Az-UV-biotin reagents, respectively, and processed as described above. In this workflow, the light to heavy ratio calculated for each WYneN-labeled cysteine residue provides a measure of its relative level in $\mathrm{H}_{2} \mathrm{O}_{2}$-treated samples versus unoxidized control 
samples. In total, we identified and quantified 2,234 probe-modified sites on 1,633 proteins, including 200 numerous functionally important cysteine residues (Supplementary Fig. 13a and Supplementary Table 201 3). Of these, $9.9 \%$ quantified sites showed $\geq 1.5$-fold dynamic changes after $\mathrm{H}_{2} \mathrm{O}_{2}$ treatment. Positively 202 regulated S-sulfenylation sites likely indicate protein-stabilized sulfenic acids, while negatively regulated 203 S-sulfenylation sites suggests overoxidation. For example, S-sulfenylation of the active sites of PRDX6 204 (C47) and $\mathrm{ASAH} 1$ (C43) decreased upon $\mathrm{H}_{2} \mathrm{O}_{2}$ treatment, indicative of hyperoxidation to sulfinic and 205 sulfonic acid states (Supplementary Fig. 13b). Moreover, S-sulfenylation of the surface-exposed cysteine 206 residue $\mathrm{C} 152$ in GAPDH was negatively regulated at a ratio of 0.88 , in contrast, the buried cysteine C247 207 showed a 2.44-fold sulfenylation under $\mathrm{H}_{2} \mathrm{O}_{2}$-induced stress (Supplementary Fig. 13b). Likewise, three 208 cysteine residues C90, C152 and C220 of ubiquitin carboxy-terminal hydrolase L1 (UCHL1) were all 209 identified as S-sulfenylation sites, and the most-buried, non-catalytic C220 showed a 2.6-fold increase of 210 modification under stress (Supplementary Fig. 13b). This residue was previously found for UCHL1 to 211 promote the assembly of mTOR complex 2 and phosphorylation of the pro-survival kinase AKT and is a 212 known S-nitrosylation site as well as a potential alkylation site $24-26$. These findings reinforce the concept 213 of dynamic protein S-sulfenylation in cells and offer hypotheses to explain how non-catalytic cysteines 214 may affect enzymatic functions via redox regulation.

216 Upon deeper analysis of these data, we noted that WYneN detected sulfenic acid modification of the key 217 nucleophilic cysteine within redox-active CXXC motifs ${ }^{27}$ (Fig. 3h). The "attacking" cysteines in this 218 sequence are susceptible to rapid thiol-disulfide exchange with the "resolving" cysteine that precludes 219 chemical ligation by less efficient dimedone-based probes. By contrast, WYneN enabled dynamic 220 quantification of sulfenic acid modification at the "attacking" cysteines of many CXXC-containing proteins, 221 including C32 of thioredoxin-1 (TRX-1), C23 of glutaredoxin-1 (GRX-1), C397 of protein disulfide222 isomerase (PDIA1), and C12 of antioxidant 1 copper chaperone (ATOX-1) (Fig. 3i). The ability of WYneN 223 to effectively trap and label the sulfenic acid state of TRX-1, GRX-1 and ATOX-1 was further validated 224 by intact MS and in-gel fluorescence analyses of these recombinant proteins (Fig. 3j,k). These findings 225 reveal the CXXC motif in thiol oxidoreductases as heretofore unrecognized direct targets of oxidation. 
a

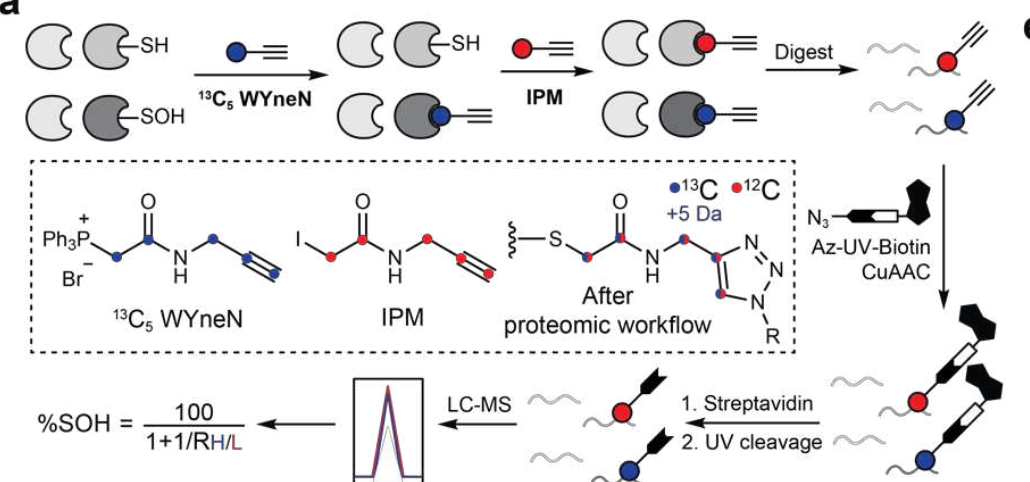

b

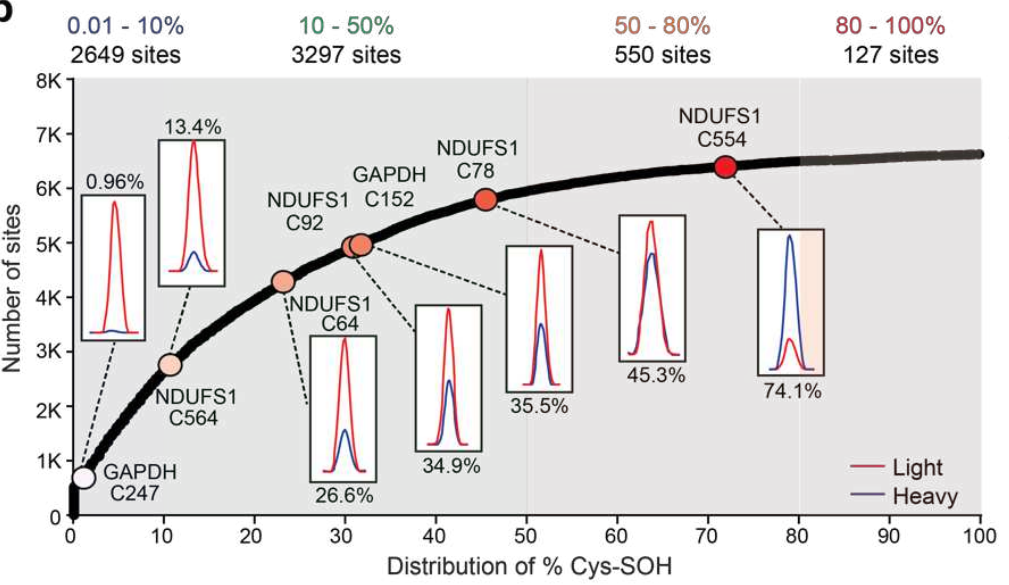

C $\% \mathrm{SOH}$

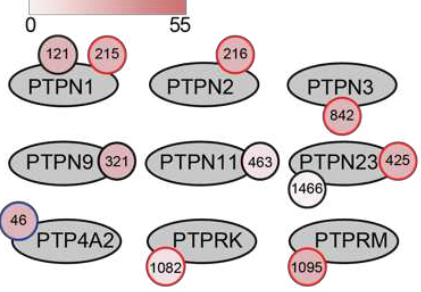

d

\section{e}

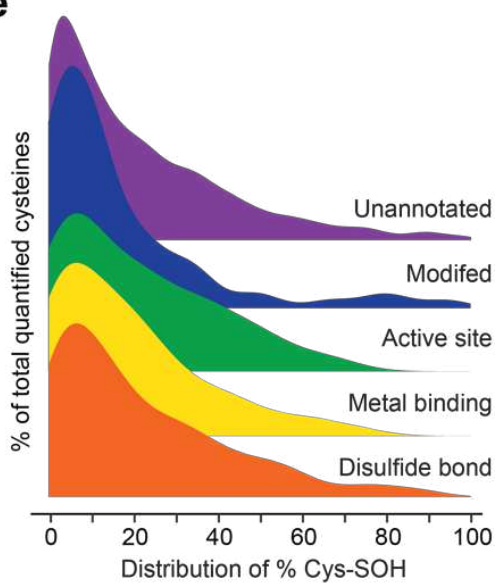

f

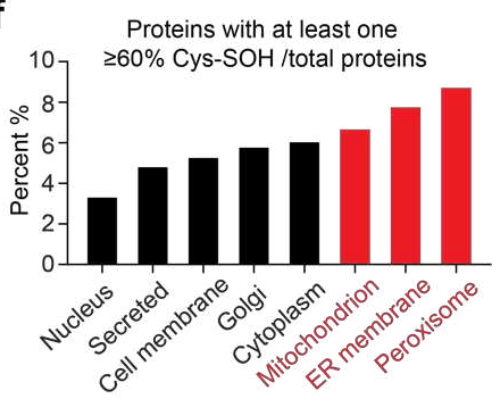

g

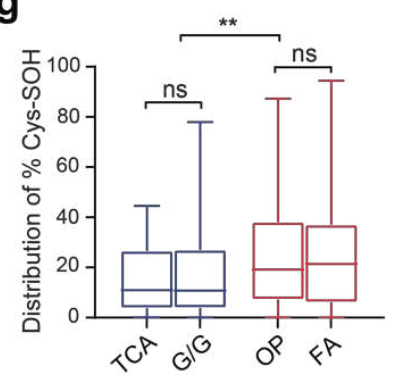

Figure 4 | Proteome-wide analysis of cysteine sulfenic acid site stoichiometry. a, Quantitative determination of site-specific occupancy of protein sulfenylation (\%SOH) in A549 cells. b, Distribution of the \%SOH values of 6,623 cysteines, which are classified into four groups as indicated. Insets: representative XICs showing for the relative levels of $\mathrm{SOH}$ (labeled with ${ }^{13} \mathrm{C}_{5} \mathrm{WYneN}$ ) and SH (labeled with IPM) in cysteines from NDUFS1 and GAPDH. c-d, \%SOH of cysteines in protein tyrosine phosphatases (c) and peroxiredoxins (d). e, Density plots showing the distribution of \%SOH in cysteines with or without functional annotations. f, Bar chart showing the distribution of proteins of high oxidation group (Site occupancy $\geq 60 \%$ ) according to subcellular locations retrieved UniProt. g, Box plot showing the \%SOH distribution of cysteines within proteins involved in selected gene ontology biological processes. TCA: tricarboxylic acid cycle, G/G: glycolysis/gluconeogenesis, OP: oxidative phosphorylation, FA: fatty acid $\beta$-oxidation. $\mathrm{P}$ values were calculated using a two-tailed, unpaired t-test. ns, not significant, ${ }^{* *} \mathrm{P} \leq 0.01$.

Proteome-wide analysis of cysteine sulfenic acid site stoichiometry. Although methods have been 
233 the mechanistic implications of cysteine oxidation in regulating protein function and targeted covalent 234 drug pharmacology. In the course of our chemoproteomic experiments with WYneN, we recognized that 235 the TPP-cleaved sulfenic acid modification was identical to that resulting from the reaction of a thiol and 236 the alkyne-tagged iodoacetamide probe, IPM (Fig. 1d) ${ }^{28}$. On the basis of this observation, we reasoned 237 that these two probes - WYneN and IPM - could generate chemically identical proteins/peptides but differ 238 in the isotopic mass of their label depending on the original redox state of the cysteine residue. To test 239 this possibility, we synthesized the heavy isotopomer, ${ }^{13} \mathrm{C}_{5} \mathrm{WYneN}$, and elaborated a chemoproteomic 240 workflow to quantify site-specific S-sulfenylation stoichiometry (Fig. 4a). First, sulfenic acids were labeled 241 in situ by incubating cells with ${ }^{13} \mathrm{C}_{5} \mathrm{WYneN}$. Next, native lysates were generated from the ${ }^{13} \mathrm{C}_{5} \mathrm{WYneN}-$ 242 treated cells and reacted with the thiol-reactive probe, IPM. The isotopically labeled samples were then 243 processed and analyzed using the aforementioned chemoproteomic workflow. Site-specific sulfenic acid stoichiometry (\%SOH) was calculated using the equation $100 /\left(1+1 / R_{H / L}\right)$.

A total of 7,357 probe-modified peptides were identified and quantified, enabling quantification of $\% \mathrm{SOH}$ values on 6,623 unique cysteine residues on 3,372 proteins (Supplementary Table 4). Consistent with the often-transient nature of sulfenic acid in cells, the \%SOH values for the majority of the cysteinome (73\%) were calculated to be lower than $30 \%$, with an average of $21.1 \%$ and a median of $14.5 \%$ (Fig. $4 b$ ). We also found that multiple cysteines on the same protein had significantly different \%SOH values. For example, five sulfenylated cysteines were mapped onto NDUFS1, a core subunit of the mitochondrial complex I, with $\% \mathrm{SOH}$ values ranging from $13.4 \%$ to $74.1 \%$ (Fig. $4 \mathrm{~b}$ ), among which were three metal binding sites (C64, C78 and C92). In another case, \%SOH value of $\mathrm{C} 152$ on GAPDH was found to be much higher than that of C247 (Fig. 4b), in accordance with our previous finding based on spectral counting of sulfenylated peptides bearing these two sites ${ }^{13}$. Also of interest, we measured \%SOH values for many protein tyrosine phosphatases (PTPs), which contain a conserved [I/V]HCSXGXGR[S/T]G motif 257 in their active site (Fig. 4c). The invariant cysteine is essential for catalysis and can be negatively 258 regulated by oxidation ${ }^{29}$. Such active sites in the majority of PTPs showed a higher \%SOH value than 259 the median value for overall sites (Fig. 4b,c). For example, we detected $20.0 \%$ S-sulfenylation of PTPN1 
C215 (also known as PTP1B), a value remarkably similar to the \% reversible oxidation determined using

261 targeted MS approaches ${ }^{30}$. In yet another case, we noted that the active site of 1-Cys peroxiredoxin 262 PRDX6 (C47) showed a relatively high \%SOH value (36.5\%), compared to the peroxidatic or resolving 263 cysteines of 2-Cys peroxiredoxins (Fig. 4d). This finding is logical given that PRDX6 is less prone to 264 disulfide bond formation compared to 2-Cys peroxiredoxins ${ }^{31}$.

In order to investigate the relationship between $\% \mathrm{SOH}$ and functionality, we retrieved information about cysteine residues with annotated functions from the UniProt knowledge database ${ }^{32}$. In general, modified cysteines (mainly through S-nitrosylation) tended to be less S-sulfenylated than other annotated or unannotated cysteines (Fig. 4e), as different types of cysteine modifications compete for the same site, thereby diminishing the $\% \mathrm{SOH}$. By contrast, active-site cysteines were distributed more broadly in the

271 range of $30-60 \%$ S-sulfenylation, compared to those with other functional annotations or without annotation (Fig. 4e). In addition to PTPs, other classes of enzymes in which the active site cysteine is highly prone to oxidation and known to be redox regulated, such as ubiquitinating and deubiquitinating enzymes, were identified ${ }^{33}$ (Supplementary Table 4). We also examined the cellular localization and Gene Ontology (GO) classification of the proteins with cysteines within the different ranges of \%SOH (Fig. 4f,g). Major oxidant-generating cellular compartments, including the peroxisome, endoplasmic reticulum and mitochondrion, were distinguished as having more highly S-sulfenylated proteins (\%SOH $\geq 60$ ) as compared to other compartments (Fig. 4f). Likewise, protein cysteines involved in two key oxidative pathways, oxidative phosphorylation and fatty acid oxidation, exhibited significantly higher overall \%SOH relative to other metabolic pathways, including the TCA cycle and glycolysis/gluconeogenesis pathways (Fig. $4 \mathrm{~g}$ ). Going forward, quantification of \%SOH using the ${ }^{13} \mathrm{C}_{5}$ WYneN probe for selective ligation will greatly assist in prioritizing sites for functional analyses and defining mechanistic models of redox-dependent protein regulation.

Imaging redox-dependent changes in mitochondrial cysteine oxidation. Organelles play a critical 

and TPP is one of the most prominent examples (Fig. 1e) . $^{35}$.

a

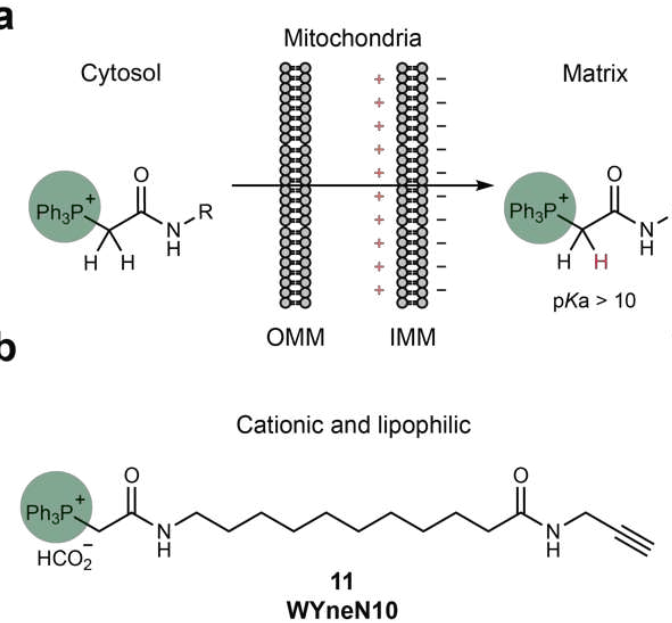

d

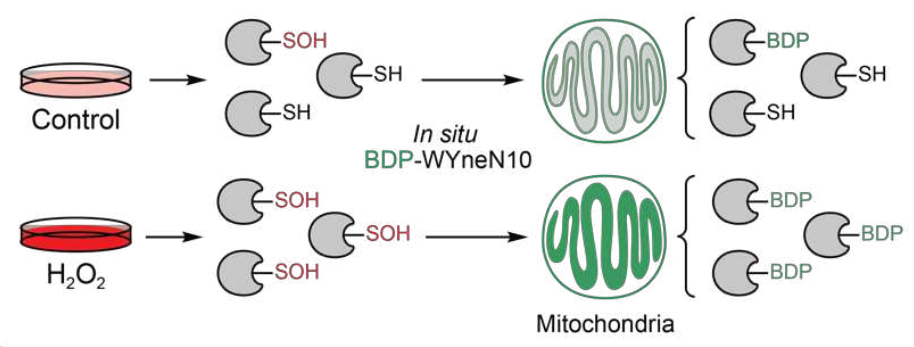

f
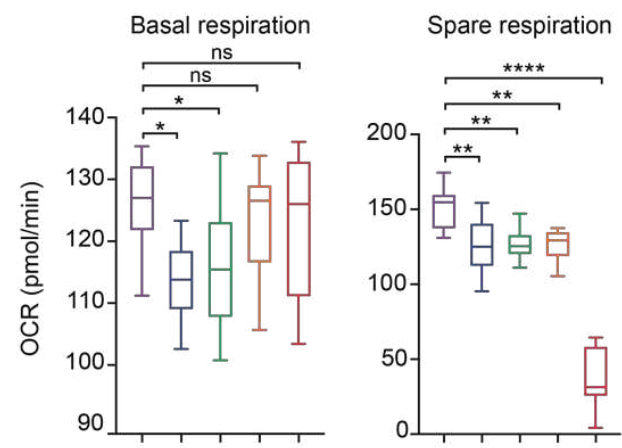

Maximal respiration
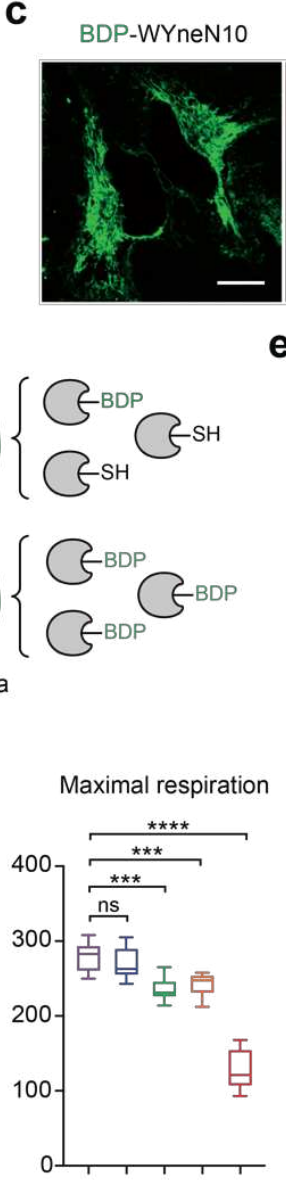

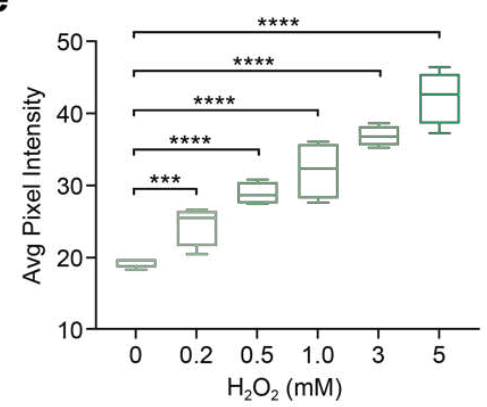

MitoTracker

Merge $(R=0.85)$
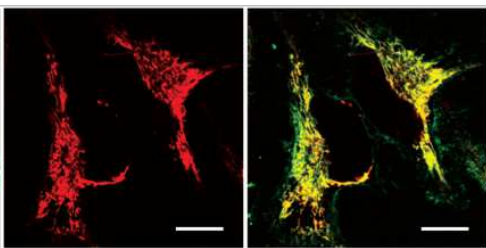

e

ATP synthesis

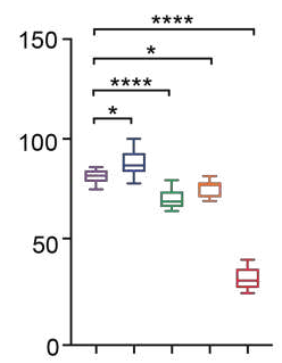

$\square$ Control

$\square$ WYneC

$\square$ WYneO

$\square$ WYneN

$\square$ WYneN10

Figure 5 | Imaging redox-dependent changes in mitochondrial cysteine oxidation. a, Amide derivative of Wittig reagents exists predominantly in protonated form, setting stage for enrichment and detecting S-sulfenylation in mitochondria. b, Structure of the mitochondrial targeting sulfenic acid probe WYneN10 with enhanced lipophilicity. c, Live cell imaging of BDP-WYneN10 suggested its mitochondrial localization in HeLa cells. MitoTracker: MitoTracker ${ }^{\mathrm{TM}}$ Deep Red FM; R, Pearson's correlation coefficient. A scale bar of $20 \mu \mathrm{m}$ is shown. d, BDP-WYneN10 tagged S-sulfenylated proteins with fluorescence inside mitochondria. e, BDP-WYneN10 fluorescence responded to external oxidative stress $\left(0-5 \mathrm{mM} \mathrm{H}_{2} \mathrm{O}_{2}\right)$ in live A549 cells $(n=4)$. f, WYneN10 disrupted mitochondrial respiration in A549 cells to a greater extent than other WYne probes. OCR, oxygen consumption rate. Error bars represent \pm s.e.m of biological replicates $(n=6)$. P values were calculated using a two-tailed, unpaired t-test. ns, not significant, ${ }^{*} P \leq 0.05,{ }^{* *} P \leq 0.01,{ }^{* \star *} P \leq 0.001,{ }^{* \star * *} P \leq 0.0001$. 
294 Reminiscent of the TPP-targeting moiety, WYneN $\left(p K_{a}>10\right)$ is predominantly protonated at physiological $295 \mathrm{pH}$. Despite its lower abundance, the deprotonated form of WYneN remains highly reactive with sulfenic 296 acid. Given the $\mathrm{pH}$ gradient of approximately one unit between the mitochondrial matrix $(\mathrm{pH}=8)$ and the 297 cytosol $(\mathrm{pH}=7)^{36}$, we hypothesized that WYneN would partition to the mitochondria, become more 298 deprotonated, and preferentially label protein sulfenic acids in this compartment (Fig. 5a). To test this 299 idea, we functionalized WYneN with a BODIPY tag for fluorescence visualization, but live cell imaging 300 suggested a poor localization of fluorescence $(R<0.3)$. This finding is consistent with the lack of 301 compartmental bias observed in our chemoproteomic studies and could be explained by the ionic nature 302 of WYneN, which decreases its inherent ability to penetrate phospholipid membranes. To tackle this 303 issue, we synthesized a WYneN derivative with a 10-carbon aliphatic linker, WYneN10 (11, Fig. 5b). 304 Satisfyingly, BODIPY-tagged WYneN10 (BDP-WYneN10) exhibited robust colocalization with the 305 commercial mitochondria indicator, MitoTracker Deep Red in live A549, HeLa, RKO, and NIH3T3 cells, 306 indicating probe accumulation in this organelle (Fig. 4c and Supplementary Fig. 14). In addition, 307 mitochondrial uncouplers such as carbonyl cyanide 4-(trifluoromethoxy)phenylhydrazone (FCCP) and 308 antimycin A dissipated mitochondrial membrane potential, and suppressed the mitochondrial staining 309 pattern (Supplementary Fig. 15). Next, we tested if BDP-WYneN10 could detect redox-dependent 310 changes in mitochondrial cysteine oxidation - using sulfenic acid content as an indicator - under 311 conditions of exogenous oxidative stress. Live A549 cells were imaged after treatment of BDP-WYneN10 312 and $\mathrm{H}_{2} \mathrm{O}_{2}$. Indeed, mitochondrial BODIPY fluorescence intensity increased concomitant with oxidant 313 concentration (Fig. 5d,e and Supplementary Fig. 16) that can be attributed to increased sulfenic acid 314 modification of mitochondrial proteins concomitant with covalent reaction of BDP-WYneN10. To further 315 characterize the effect of WYne probes on mitochondria function, we performed a mitochondrial stress 316 test and monitored the oxygen consumption rate (OCR) of A549 cells. Of all WYne probes, only lipophilic, 317 cationic WYneN10 disrupted mitochondrial respiration with respect to spare respiratory capacity, maximal 318 respiration and ATP production (Fig. 5f and Supplementary Fig. 17). Overall, these data indicate the 319 elevated $\mathrm{p} K_{\mathrm{a}}$ inherent to the amide-functionalized lipophilic Wittig reagent, WYneN10, and its reactivity 320 with sulfenic acid, can be exploited to visualize changes in mitochondrial cysteine oxidation. 
a

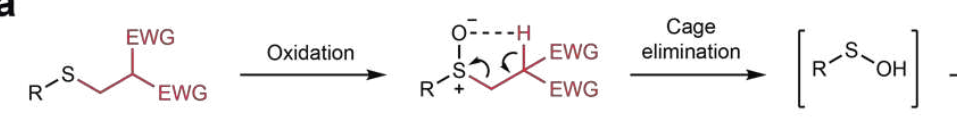

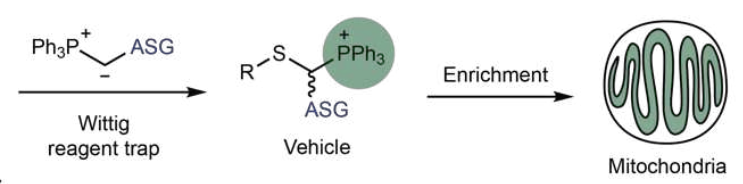

b<smiles>COC(=O)C(NC(=O)[C@H](CSC(C(C)=O)C(C)C)C(=O)NCc1ccccc1)C(C)C</smiles>

Vehicle 15

BDP

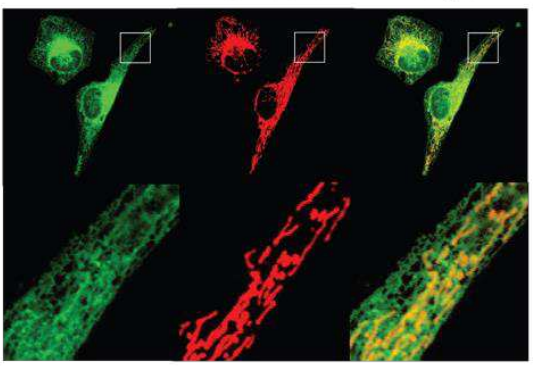

$R=0.55$<smiles>CCOC(=O)C(CSC[C@H](NC(=O)c1ccccc1)C(=O)NC(=O)c1ccccc1)C(C)C</smiles>

Vehicle 16<smiles>COC(=O)[C@@H](NC(=O)[C@H](CSSC[C@H](NC(=O)c1ccccc1)C(=O)N[C@H](C(=O)OC)C(C)C)NC(=O)c1ccccc1)C(C)C</smiles>

Disulfide 18
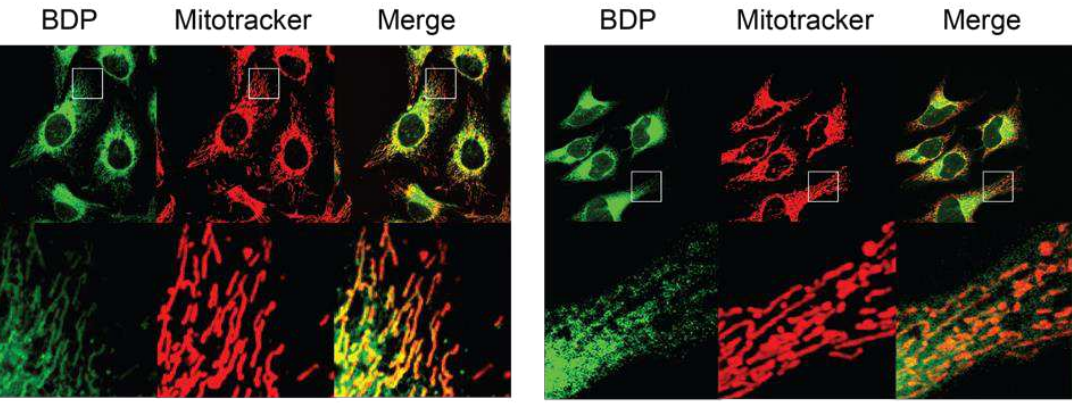

$R=0.80$

$R=0.39$

C

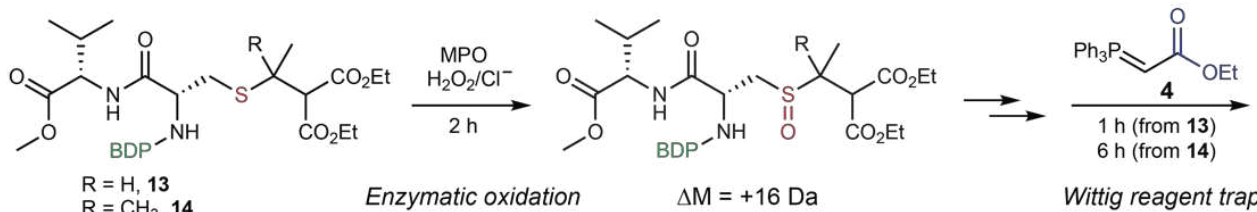
$\mathrm{R}=\mathrm{CH}_{3}, 14$

Enzymatic oxidation

$\Delta \mathrm{M}=+16 \mathrm{Da}$<smiles>CCOC(=O)C([18O])([Pb])SC[C@H](NC(=O)c1ccccc1)C(=O)N[C@H](C(=O)OC)C(C)C</smiles>

Wittig reagent trap

d

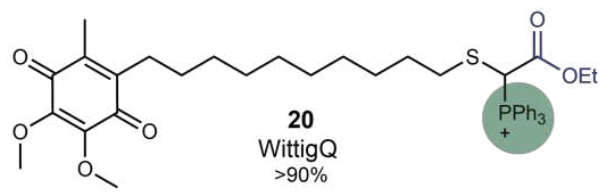
$\underset{\text { Complex II activity }}{\stackrel{\text { Isolated mitochondria }}{\longrightarrow}}$ $2 \mathrm{H}^{+}+2 \mathrm{e}^{-}$

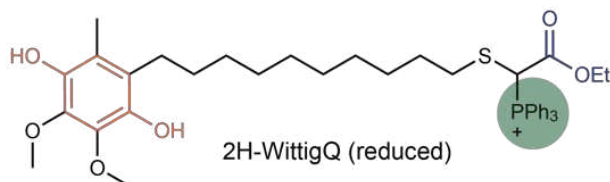

e

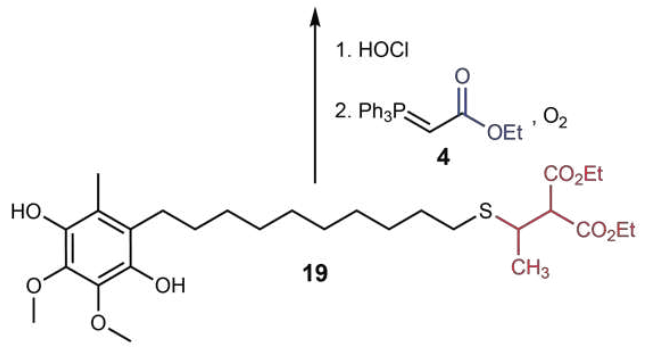
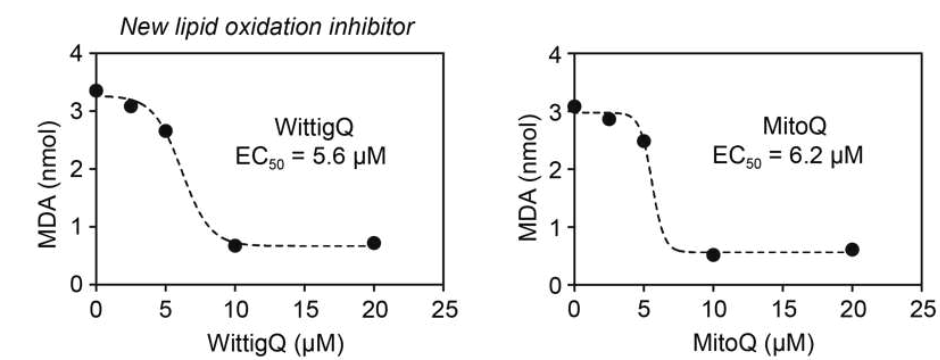

Figure 6 | Redox-triggered in situ TPP generation for mitochondrial cargo delivery. a, General principle of a redox-caged mitochondrial targeting system. b, Comparison of two different vehicle and a disulfide control compound in live cell imaging. BDP, BODIPY-tagged mitochondrial vehicles; MitoTracker: MitoTracker ${ }^{\mathrm{TM}}$ Deep Red FM; R, Pearson's correlation coefficient calculated as the average of five regions of interest (ROls) from representative cells. A zoom-in area of $20 \times 20 \mu \mathrm{m}$ was shown below each image. c, Enzymatic activation of the redoxcaged sulfenic acids $\mathbf{1 3}$ and $\mathbf{1 4}$ by generation of $\mathrm{HOCl}$ from myeloperoxidase (MPO), followed by Wittig reagent trapping. d, Redox-caged sulfenic acid 19 was oxidized by $\mathrm{HOCl}$, reacted with Wittig reagent 4 to afford the mitochondria-targeting compound, WittigQ (20), which was actively reduced by complex II activity to the quinol form. e, WittigQ prevented lipid peroxidation in mitochondria isolated from HeLa cells at comparable efficacy to MitoQ. Representative data from two independent experiments are shown.

Redox-triggered in situ TPP generation for mitochondrial delivery. To further showcase the 
324 the ligation generates a cationic TPP moiety that could be applied for mitochondrial targeting. 325 Specifically, we envisioned a system which, once triggered, generates sulfenic acid available for ligation 326 with functionalized Wittig reagents to deliver cargo inside mitochondria (Fig. 6a). To the best of our 327 knowledge, such a controllable system has not been reported, whilst the closest precedence to this 328 concept are some commercially available MitoTracker probes that are readily oxidized during basal 329 cellular respiration to become cationic and fluorescent. In contrast to existing mitochondrial targeting 330 strategies that are limited to constitutive, uncontrolled delivery ${ }^{35}$, our approach provides a reaction-based 331 switch to sequester non-cationic substrates for mitochondrial enrichment that can respond to cellular 332 redox changes.

Table 2 | Surveying sulfoxides as caged precursors to sulfenic acids and Wittig reagent trap.
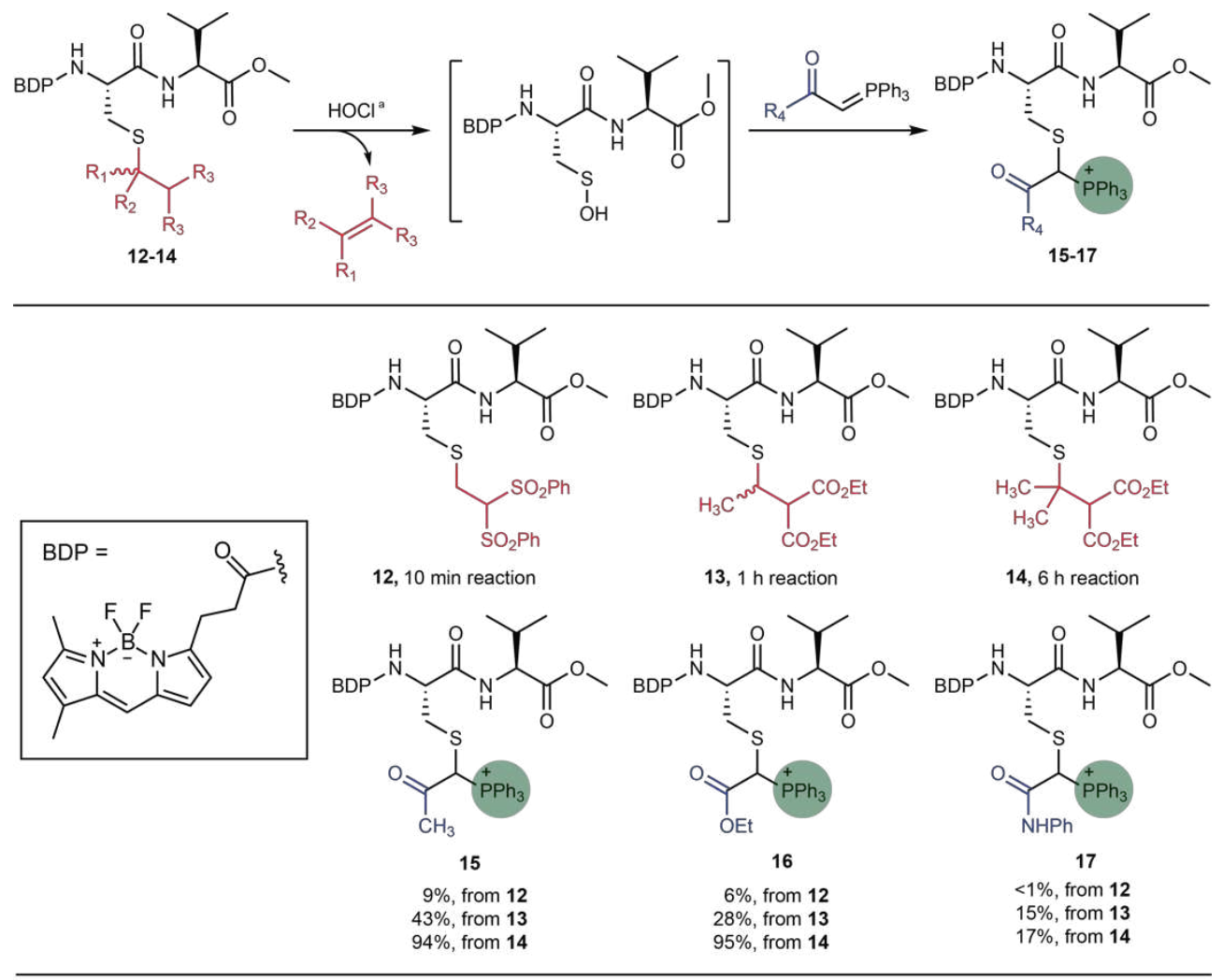

a $\mathrm{HOCl}(1 \mathrm{eq})$ was added to inititate this one-pot, two-step reaction.

334 To investigate the feasibility of this concept, we utilized sulfoxides as caged precursors to small molecule 335 sulfenic acids (Table 2). This decaging reaction typically requires heat but can take place at ambient 336 temperatures when the $\beta$-carbon is connected to strong electron withdrawing groups (EWGs) ${ }^{37,38}$. To 
study the effect of EWGs, we prepared several thioether precursors (12-14), and used hypochlorous acid

$338(\mathrm{HOCl})$ - a powerful oxidant produced by biological systems as an immune response - to convert them 339 to sulfoxides. The reaction was hindered when a methyl group was present at the $\alpha$-position and 340 decreased further with dimethyl substitution. Strength of the EWG also played an essential role. For 341 example, $\beta$-hydrogen acidity was greatly enhanced by benzenesulfonyl groups, resulting in faster 342 elimination compared to compounds with ester substituents. This series of compounds offered us a 343 broad range of rates for the decaging of sulfenic acids, from $10 \mathrm{~min}$ to $6 \mathrm{~h}$ for reaction to finish. Next, we 344 employed Wittig reagents 3-5 to capture the nascent sulfenic acids and furnish TPP derivatives 15-17. 345 The best yield was obtained from 14 with highly nucleophilic Wittig reagents 3 and $\mathbf{4}$, while cages 12 and 34613 with faster release rates gave moderate to poor yield (Table 2). Live cell images of HeLa cells co347 treated with MitoTracker and 15 or 16 indicated that the TPP-linked compounds successfully 348 accumulated in mitochondria, as opposed to a disulfide control (Fig. 6b). Ester derivative 16 exhibited a 349 higher degree of mitochondrial enrichment (Pearson's correlation coefficient $R=0.80$ ) relative to the 350 ketone derivative $15(\mathrm{R}=0.55)$, owing to the higher $\mathrm{p} K_{\mathrm{a}}$ of the ester ${ }^{39}$, which leads to a greater degree 351 of protonation at physiological pH (Supplementary Fig. 18). Enzymatically generated $\mathrm{HOCl}$ produced by 352 the myeloperoxidase (MPO) enzyme system, also provided the desired product 16 (Fig. 6c and 353 Supplementary Fig. 19). This concept was also evaluated in situ, where redox-caged sulfenic acid 13 354 was oxidized by HOCl from supplemental MPO to HeLa cells, or endogenous MPO generated by LPS355 stimulation of RAW 264.7 cells, followed by reaction with Wittig reagent 4 to afford mitochondrial vector 356 16. Live cell imaging showed elevated mitochondrial localization compared to the control which lacked 357 the $\mathrm{HOCl}$-generating MPO redox trigger (Supplementary Figs. 20 and 21). Taken together, this approach 358 offers flexible choices for redox-triggered in situ TPP functionalization and cargo delivery to mitochondria.

360 As another demonstration of our mitochondria targeting strategy, we selected the benzoquinone core of 361 coenzyme Q10 as a functional cargo. Coenzyme Q10 (ubiquinone), which is primarily reduced to 362 ubiquinol by the mitochondrial electron transport chain, has radical quenching activity through the 363 ubisemiquinone intermediate, and helps regenerate another essential antioxidant, vitamin E ${ }^{40}$. A TPP- 
substituted derivative of Coenzyme Q10, MitoQ, among many other TPP-linked antioxidants, have been developed to mitigate oxidative stress in mitochondria ${ }^{40}$. A coenzyme Q10 derivative 19 with a redoxcaged sulfenic acid moiety was prepared, and, when exposed to $\mathrm{HOCl}$, reacted with Wittig reagent 4 to give TPP-linked compound WittigQ (20, Fig. 6d). In live HeLa cells, a fluorescent WittigQ derivative showed mitochondrial localization which can be dissipated by FCCP (Supplementary Fig. 22). Experiments with isolated mitochondria further show that WittigQ functions similarly to MitoQ with respect to redox cycling and protection against lipid peroxidation (Fig. 6d,e and Supplementary Fig. 23).

\section{Discussion}

373 Herein, we described Wittig reagents as next-generation probes for protein sulfenic acids, featuring three 374 key properties: First, the probes display high nucleophilicity under physiological conditions, offering fast reaction kinetics while precluding cross-reactivity in biological systems ${ }^{16}$. Second, both the probes and their reaction products are compatible with aqueous media, as well as conditions for downstream protein analyses. Third, the carbon-based nucleophilic probes benefit from the strong C-S bond (dissociation energy $D^{\circ}{ }_{298}=712 \mathrm{~kJ} / \mathrm{mol}$ ), an advantage over heteroatom-based nucleophiles, such as amines or thiols $\left(\mathrm{N}-\mathrm{S} \text { bond } D^{\circ}{ }_{298}=467 \mathrm{~kJ} / \mathrm{mol} \text {, S-S bond } D_{298}^{\circ}=425 \mathrm{~kJ} / \mathrm{mol}\right)^{41}$. By harnessing this new chemistry, we develop a Wittig reagent-based toolbox that offers excellent kinetics and selectivity for robust sensing of protein cysteine sulfenylation in native environments and, more broadly, opens the door to chemical functionalization of biological targets in living cells.

To date, site-specific chemoproteomic profiling of protein sulfenic acids in intact cells has been predicated almost exclusively on DYn-2, a 1,3-cyclohexadione-based probe, which is quite selective, but hampered by slow reaction kinetics (Supplementary Fig. 1c). In this regard, a very large amount of protein materials (30-40 mg) is typically required ${ }^{20}$, thereby greatly precluding many physiologically relevant applications. Notably, as compared to our previous analysis using DYn- $2^{13}, 10$-fold fewer starting materials were needed for our chemoproteomic study with WYneN that achieves even a higher coverage of the sulfenylome. Strikingly, among 1,000 newly discovered in situ sulfenylated sites were the "attacking" 
cysteines within CXXC motifs that are prone to rapid thiol-disulfide exchange, further demonstrating excellent kinetics of WYne reagents. Moreover, of interest, our dose-dependent labeling experiment (Fig. 3f) reveals that the reactivity of WYneN towards sulfenic acids positively associates with functional importance (e.g., active site, disulfide, metal binding). Indeed, S-sulfenylation, as an electrophilic modification of the cysteine proteome, can effectively inverse the polarity of the nucleophilic sulfur atom and render it more reactive. Therefore, our reaction-based approach for profiling this electrophilic cysteinome directly and reliably discovers functional, redox regulated cysteines.

Quantification of \%SOH may also be essential to assess potential of drug resistance for those covalent inhibitors targeting free thiols. In addition, stoichiometric quantification of \%SOH on a particular cysteine 401 site offers another interesting aspect to identify functional nodes through which endogenous reactive oxygen species exerts regulatory functions. Existing proteomic approaches often report percent cysteine modification but rely on differential alkyation-reduction strategies to indirectly assess the total reversible oxidation, lumping all S-modifications together, in contrast with direct measurement of distinct oxoforms in the cysteinome. Using ${ }^{13} \mathrm{C}_{5} \mathrm{WYneN}$ and IPM, we report here the first measurement of \%SOH in cysteines on a proteome-wide scale in cells. Notably, many cysteine residues that play major regulatory roles in redox signaling are sulfenylated at a relatively low extent, including those in 2-Cys peroxiredoxins. In concert with this observation, a recent report demonstrates that a redox switch with low percent oxidation is indeed critical for the physiological roles of $\mathrm{Akt}^{42}$. Thus, we reason that, like other posttranslational modifications, such as phosphorylation ${ }^{43}$ and acetylation ${ }^{44}$, cysteine sulfenylation may not 411 need to reach a very high site-occupancy to exert regulatory effects.

413 Another aspect of this study was to investigate the potential of the Wittig reaction as a source of chemical 414 tools with enhanced organelle specificity. Resolution within cellular compartments is desirable because 415 many oxidants have sub-cellular distances of diffusion $(0.02 \mu \mathrm{m}$ for $\bullet \mathrm{OH}, 0.3 \mu \mathrm{m}$ for $\mathrm{HOCl}, 0.4 \mu \mathrm{m}$ for $416 \mathrm{NO}_{2}$, and $\mathrm{H}_{2} \mathrm{O}_{2}$ has a shortened range of several microns in the presence of peroxiredoxin-2, whereas 417 the typical diameter of a cell is $\sim 20 \mu \mathrm{m})^{45}$, and certain organelles (e.g., mitochondrion, peroxisome, and 
418 endoplasmic reticulum) produce more oxidants due to their functions in respiration, enzymatic activity 419 and protein folding. Of these, cysteine-mediated redox signaling in the mitochondria is key to 420 physiological processes and pathological damage within most mammalian cells ${ }^{46}$. The WYneN10 probe, 421 engineered to be cationic and lipophilic under physiological conditions, readily accumulates in 422 mitochondria and was the only compound to affect mitochondrial respiration among other Wittig probes. 423 Its BODIPY conjugate enables us to visualize changes in protein S-sulfenylation levels concomitant with 424 changes in redox homeostasis. The observation that a relatively high level (millimolar range) of 425 exogenously applied $\mathrm{H}_{2} \mathrm{O}_{2}$ was required to elicit a significant increase in fluorescence is fully consistent 426 with the floodgate hypothesis ${ }^{47}$, where overoxidation of peroxiredoxins inactivates these antioxidant 427 enzymes and permits oxidation of other protein targets.

429 More broadly, we applied Wittig reagents toward the development of controllable tools for redox biology. 430 An oxidant-triggered chemical switch for mitochondrial delivery has many advantages including improved 431 target selectivity and decreased cargo toxicity. By tuning the substituents on the redox-caged substrate, 432 we achieved a wide range of temporal responses for both acute release and prolonged actions. Beyond 433 a proof-of-concept fluorescent cargo, we implemented an antioxidant-based mitochondrial delivery 434 system to counteract the effect of endogenous $\mathrm{HOCl}$. Since $\mathrm{HOCl}$ is mainly secreted in myeloperoxidase 435 (MPO)-rich immune cells, such as neutrophils or macrophages, it causes inflammatory damage to 436 surrounding healthy cells. In this context, the $\mathrm{HOCl}$-triggered antioxidant may alleviate mitochondrial 437 oxidative damage caused by inflammation. This redox-triggered strategy opens door to more selective 438 therapies that target cancer, aging and degenerative diseases, which are often associated with elevated 439 oxidative stress. It could also improve the efficacy of established mitochondria-targeting approaches with 440 payloads such as antioxidants, apoptosis-inducing drugs, imaging molecules, and genetic materials.

442 As with all new technologies, it is worth noting limitations. WYneC and WYneO are potent tools for 443 bioconjugation but are less efficient in proteomic studies due to the complexity of fragmentation patterns. 444 The WYneN adduct is sensitive to prolonged incubation under strong reducing and basic conditions, so 
445 appropriate care must be taken during analyses. At present, the Wittig-based redox-switch is most easily 446 triggered by $\mathrm{HOCl}$. The ability to efficiently react with $\mathrm{H}_{2} \mathrm{O}_{2}$ would further enhance the application of our 447 controlled delivery mechanism. WYneN10 is well poised for imaging and detection of mitochondrial 448 protein sulfenic acids, but its hydrophobic linker limits application in quantitative MS. These and other 449 areas are interesting directions for further studies.

450

451 In summary, our findings demonstrate that Wittig reagents, together with a unique form of electrophilic 452 sulfur found in proteins constitute a new class of highly selective and biocompatible reactions. Projecting 453 forward, we envision several exciting areas wherein this reaction can be applied that should help address 454 fundamental questions about redox modification in the human cysteinome and, more broadly, the sulfenic 455 acid moiety as a target for covalent drugs and chemical ligation handle for mitochondrial targeting. 456 
458 Methods

459 A detailed Methods section is provided in the Supplementary Information.

460

\section{Reporting Summary}

462 Further information on research design is available in the Nature Research Reporting Summary linked to 463 this article.

464

465 Data availability

466 All data associated with this study are available in the published article and its Supplementary 467 Information.

468

469 


\section{References}

471 1. Sletten, E. M. \& Bertozzi, C. R. Bioorthogonal chemistry: fishing for selectivity in a sea of 472 functionality. Angew. Chem. Int. Ed. Engl. 48, 6974-6998 (2009).

$473 \quad$ 2. Devaraj, N. K. The future of bioorthogonal chemistry. ACS Cent. Sci. 4, 952-959 (2018).

474 3. Saxon, E. \& Bertozzi, C. R. Cell surface engineering by a modified Staudinger reaction. $475 \quad$ Science 287, 2007-2010 (2000).

476 4. Kolb, H. C., Finn, M. G. \& Sharpless, K. B. Click chemistry: diverse chemical function from a 477 few good reactions. Angew. Chemie Int. Ed. 40, 2004-2021 (2001).

$478 \quad$ 5. Maryanoff, B. E. \& Reitz, A. B. The Wittig olefination reaction and modifications involving 479 phosphoryl-stabilized carbanions. Stereochemistry, mechanism, and selected synthetic $480 \quad$ aspects. Chem. Rev. 89, 863-927 (1989).

$481 \quad 6$ El-Batta, A. et al. Wittig reactions in water media employing stabilized ylides with aldehydes. $482 \quad$ Synthesis of $\alpha, \beta$-unsaturated esters from mixing aldehydes, $\alpha$-bromoesters, and Ph3P in 483 aqueous NaHCO3. J. Org. Chem. 72, 5244-5259 (2007).

484 7. Gupta, V. \& Carroll, K. S. Sulfenic acid chemistry, detection and cellular lifetime. Biochim. 485 Biophys. Acta - Gen. Subj. 1840, 847-875 (2014).

486 8. Gupta, V. \& Carroll, K. S. Profiling the reactivity of cyclic C-nucleophiles towards 487 electrophilic sulfur in cysteine sulfenic acid. Chem. Sci. 7, 400-415 (2016).

$488 \quad$ 9. Holmström, K. M. \& Finkel, T. Cellular mechanisms and physiological consequences of 489 redox-dependent signalling. Nat. Rev. Mol. Cell Biol. 15, 411-421 (2014).

490 10. Paulsen, C. E. \& Carroll, K. S. Cysteine-mediated redox signaling: chemistry, biology, and 491 tools for discovery. Chem. Rev. 113, 4633-4679 (2013).

492 11. Sies, H. \& Jones, D. P. Reactive oxygen species (ROS) as pleiotropic physiological 493 signalling agents. Nat. Rev. Mol. Cell Biol. 21, 363-383 (2020).

$494 \quad$ 12. Leonard, S. E., Reddie, K. G. \& Carroll, K. S. Mining the thiol proteome for sulfenic acid 495 modifications reveals new targets for oxidation in cells. ACS Chem. Biol. 4, 783-799 (2009). 
496 13. Yang, J., Gupta, V., Carroll, K. S. \& Liebler, D. C. Site-specific mapping and quantification of protein S-sulphenylation in cells. Nat. Commun. 5, 4776 (2014).

14. Gupta, V. \& Carroll, K. S. Rational design of reversible and irreversible cysteine sulfenic acid-targeted linear C-nucleophiles. Chem. Commun. 52, 3414-3417 (2016).

15. Gupta, V., Yang, J., Liebler, D. C. \& Carroll, K. S. Diverse redoxome reactivity profiles of carbon nucleophiles. J. Am. Chem. Soc. 139, 5588-5595 (2017).

16. Shi, Y. \& Carroll, K. S. Activity-based sensing for site-specific proteomic analysis of cysteine oxidation. Acc. Chem. Res. 53, 20-31 (2020).

17. Poole, L. B. et al. Fluorescent and affinity-based tools to detect cysteine sulfenic acid formation in proteins. Bioconjug. Chem. 18, 2004-2017 (2007).

18. Poole, L. B., Zeng, B.-B., Knaggs, S. A., Yakubu, M. \& King, S. B. Synthesis of chemical (2005).

19. Pan, J. \& Carroll, K. S. Light-mediated sulfenic acid generation from photocaged cysteine sulfoxide. Org. Lett. 17, 6014-6017 (2015).

20. Yang, J. et al. Global, in situ, site-specific analysis of protein S-sulfenylation. Nat. Protoc. 10, 1022-1037 (2015).

21. Fu, L., Liu, K., Ferreira, R. B., Carroll, K. S. \& Yang, J. Proteome-wide analysis of cysteine S-sulfenylation using a benzothiazine-based probe. Curr. Protoc. Protein Sci. 95, e76 (2019).

22. Sun, R. et al. Chemoproteomics reveals chemical diversity and dynamics of 4-oxo-2nonenal modifications in cells. Mol. Cell. Proteomics 16, 1789 LP - 1800 (2017).

23. Chi, H. et al. Comprehensive identification of peptides in tandem mass spectra using an efficient open search engine. Nat. Biotechnol. 36, 1059-1061 (2018).

24. Hussain, S. et al. A cysteine near the C-terminus of UCH-L1 is dispensable for catalytic activity but is required to promote AKT phosphorylation, elF4F assembly, and malignant Bcell survival. Cell Death Discov. 5, 152 (2019). 
523 25. Liu, Z. et al. Membrane-associated farnesylated UCH-L1 promotes $\alpha$-synuclein neurotoxicity and is a therapeutic target for Parkinson's disease. Proc. Natt. Acad. Sci. 106, 4635-4640 (2009).

26. Kumar, R. et al. S-nitrosylation of UCHL1 induces its structural instability and promotes $\alpha$ synuclein aggregation. Sci. Rep. 7, 44558 (2017).

27. Berndt, C., Lillig, C. H. \& Holmgren, A. Thioredoxins and glutaredoxins as facilitators of protein folding. Biochim. Biophys. Acta - Mol. Cell Res. 1783, 641-650 (2008).

28. Fu, L. et al. Systematic and quantitative assessment of hydrogen peroxide reactivity with

37. Adams, $H$. et al. The synthesis and Diels-Alder reactions of (E)- and (Z)-1-methoxy-3-

29. Karisch, R. et al. Global proteomic assessment of the classical protein-tyrosine phosphatome and "redoxome". Cell 146, 826-840 (2011).

30. Lou, Y.-W. et al. Redox regulation of the protein tyrosine phosphatase PTP1B in cancer cells. FEBS J. 275, 69-88 (2008).

31. Zhou, S. et al. Peroxiredoxin 6 homodimerization and heterodimerization with glutathione Stransferase pi are required for its peroxidase but not phospholipase A2 activity. Free Radic. Biol. Med. 94, 145-156 (2016).

32. The UniProt Consortium. UniProt: the universal protein knowledgebase. Nucleic Acids Res. 45, D158-D169 (2017).

33. Pajares, M. et al. Redox control of protein degradation. Redox Biol. 6, 409-420 (2015).

34. Murphy, M. P. \& Smith, R. A. J. Targeting antioxidants to mitochondria by conjugation to lipophilic cations. Annu. Rev. Pharmacol. Toxicol. 47, 629-656 (2007).

35. Frantz, M.-C. \& Wipf, P. Mitochondria as a target in treatment. Environ. Mol. Mutagen. 51, 462-475 (2010).

36. Casey, J. R., Grinstein, S. \& Orlowski, J. Sensors and regulators of intracellular pH. Nat. Rev. Mol. Cell Biol. 11, 50-61 (2010). (phenylsulfinyl)buta-1,3-dienes. J. Chem. Soc. Perkin Trans. 13967-3974 (1998). 
550 38. Barattucci, A. et al. Transient sulfenic acids in the synthesis of biologically relevant products. Molecules 23, 1030 (2018).

39. Zhang, X. M. \& Bordwell, F. G. Equilibrium acidities and homolytic bond dissociation energies of the acidic carbon-hydrogen bonds in P-substituted triphenylphosphonium cations. J. Am. Chem. Soc. 116, 968-972 (1994).

40. Kelso, G. F. et al. Selective targeting of a redox-active ubiquinone to mitochondria within cells. J. Biol. Chem. 276, 4588-4596 (2001).

41. Luo, Y.-R. Comprehensive handbook of chemical bond energies (CRC Press, 2007).

42. Su, Z. et al. Global redox proteome and phosphoproteome analysis reveals redox switch in Akt. Nat. Commun. 10, 5486 (2019).

43. Wu, R. et al. A large-scale method to measure absolute protein phosphorylation stoichiometries. Nat. Methods 8, 677-683 (2011).

44. Hansen, B. K. et al. Analysis of human acetylation stoichiometry defines mechanistic constraints on protein regulation. Nat. Commun. 10, 1055 (2019).

45. Winterbourn, C. C. Reconciling the chemistry and biology of reactive oxygen species. Nat. Chem. Biol. 4, 278-286 (2008).

46. Bak, D. W. \& Weerapana, E. Cysteine-mediated redox signalling in the mitochondria. Mol. BioSyst. 11, 678-697 (2015).

47. Wood, Z. A., Poole, L. B. \& Karplus, P. A. Peroxiredoxin evolution and the regulation of hydrogen peroxide signaling. Science, 300, 650-653 (2003). 


\section{Acknowledgements}

572 We thank Longqin Sun and Tuo Zhang from Beijing Qinglian Biotech Co., Ltd for their help and 573 technical support. This work was supported by the US National Institutes of Health (R01 574 GM102187 and R01 CA174864 to K.S.C) and the National Natural Science Foundation of China 575 (21922702), the National Key R\&D Program of China (2016YFA0501303), and the State Key 576 Laboratory of Proteomics (SKLP-K201703 and SKLP-K201804) to J.Y.

578 Author Contributions

579 Y.S., J.Y., and K.S.C. conceived of the project, designed experiments and analyzed data. Y.S. 580 synthesized and characterized compounds. Y.S. and L.F. performed intact MS and quantitative 581 proteomic experiments and data analysis. Y.S. performed probe validation and cell-based 582 experiments.

583

584 Competing Interests statement

585 The authors declare no competing financial interests.

586

587 


\section{Figures}

a

Wittig Reagents: Originally used for alkene synthesis

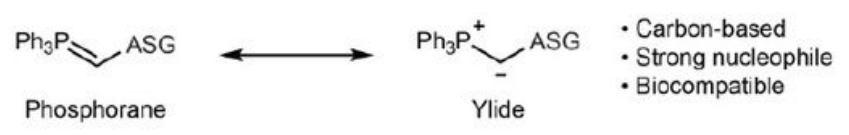

b

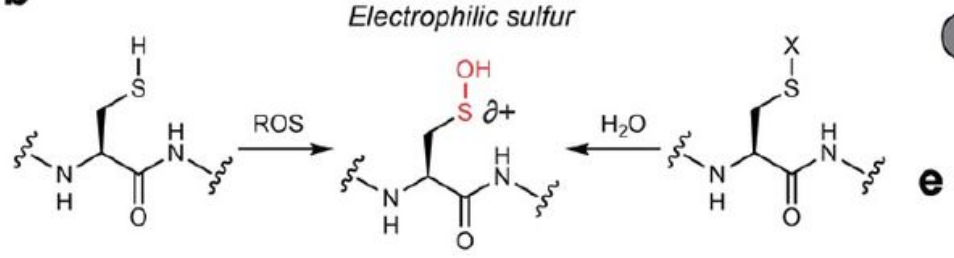

C

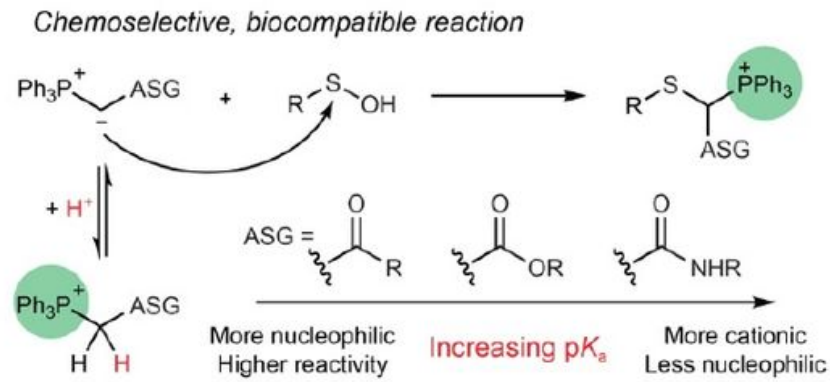

d
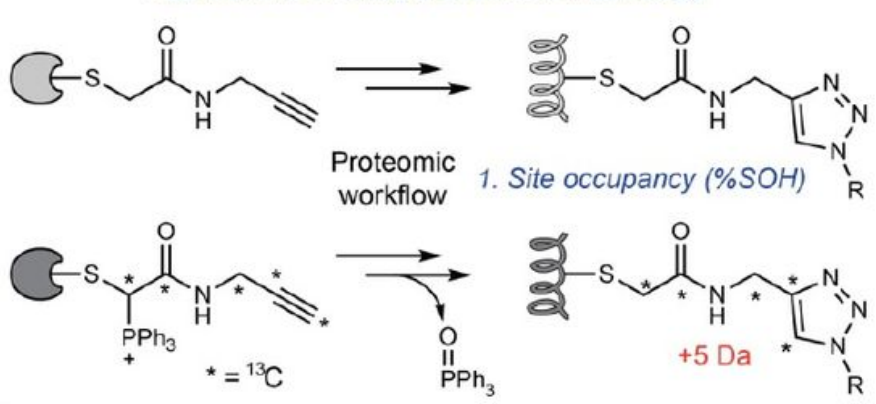

e

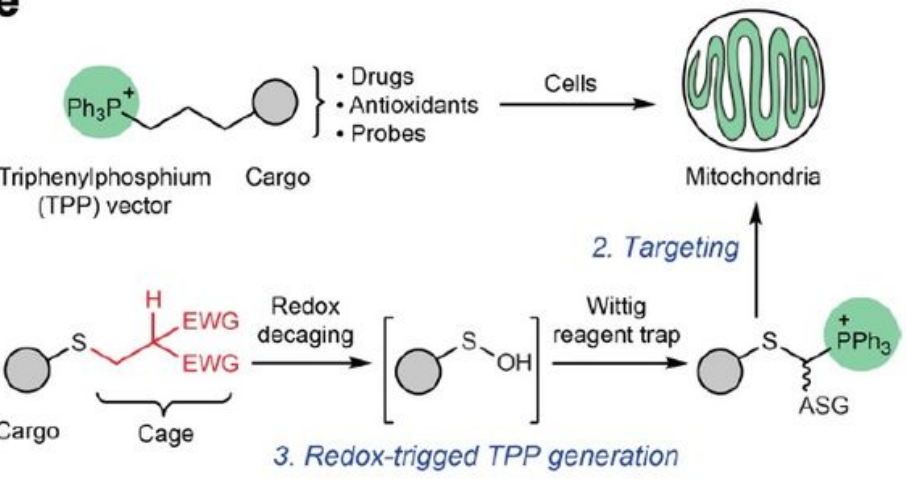

\section{Figure 1}

Repurposing triphenylphosphonium ylides as probes for electrophilic sulfur in proteins. a, Wittig reagents can be illustrated as the phosphorane or ylide form. With anion-stabilizing groups (ASG), Wittig reagents act as water-compatible carbon-based nucleophiles. b, Sulfenic acids are formed via two major pathways: direct oxidation or hydrolysis of polarized sulfur species. c, Biocompatible reaction between sulfenic acids and stabilized Wittig reagents. $d$, Determination of S-sulfenylation site occupancy. After proteomic workflow, labeled protein thiols (top) and sulfenic acids (bottom) yielded isotopomers. e, Taking advantage of the TPP group installed after reaction with the Wittig reagent enables direct or redoxtriggered cargo delivery to mitochondria. 
a

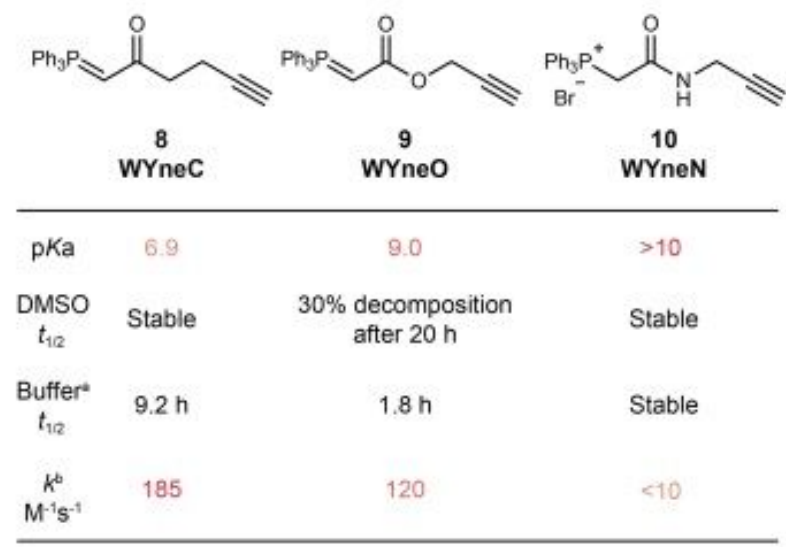

b

$\sim 50$ mM HEPES, $100 \mathrm{mM} \mathrm{NaCl}$, pH $=7.4 \quad$ "ACN:NaOAC (1:2) pH = 4.9

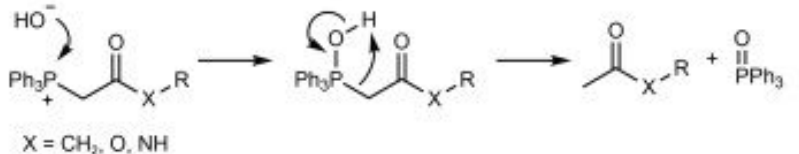

C

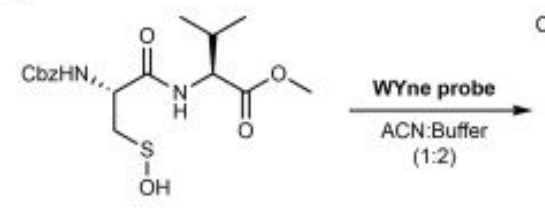

Dipeptide-SOH

d

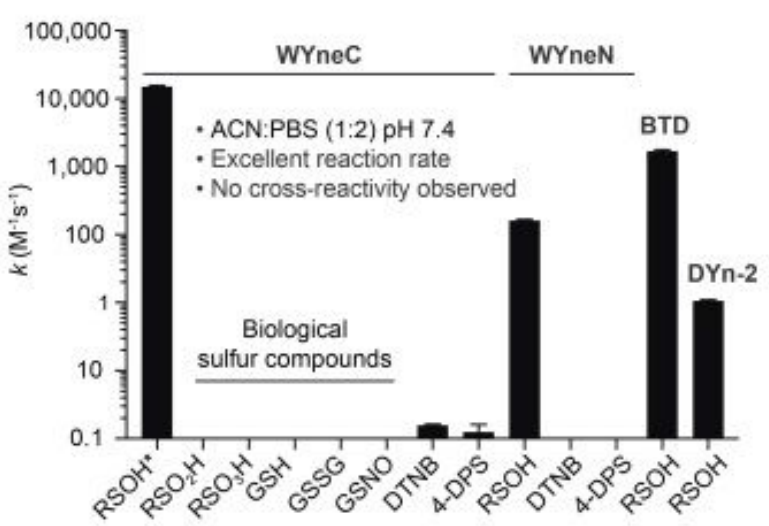

e s. cerevisiae Gp $\times 3$
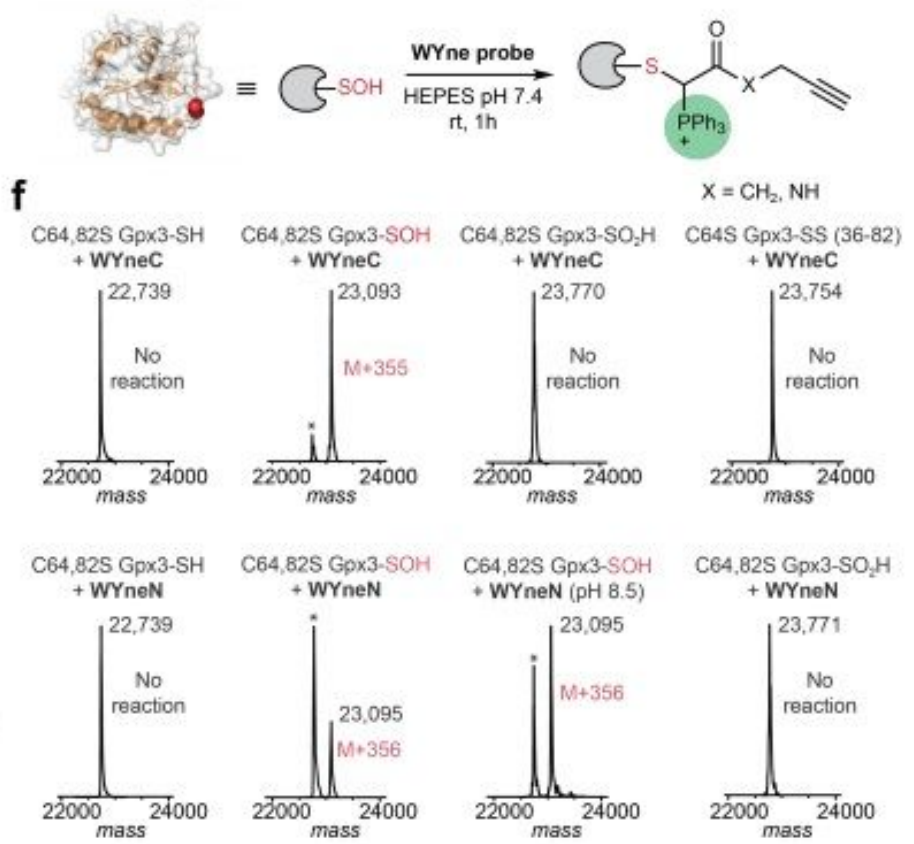

C64,82S Gpx3-SO, $\mathrm{H}$

+ WYneN
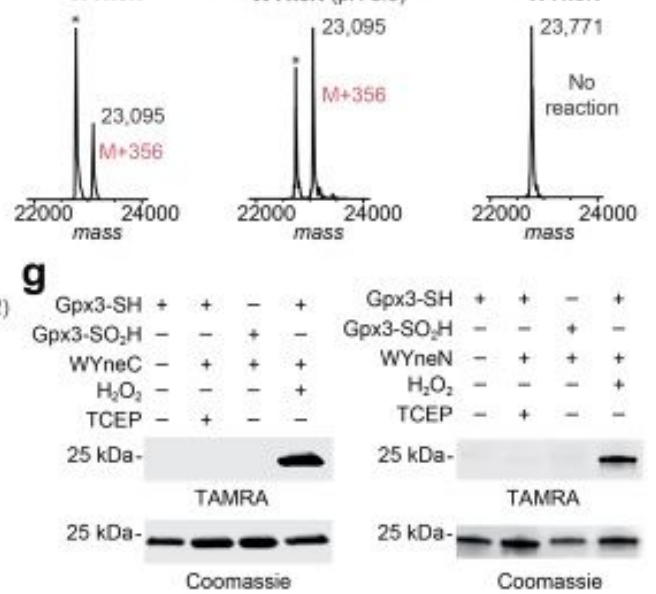

h

C64S Gpx3-SS (36-82)
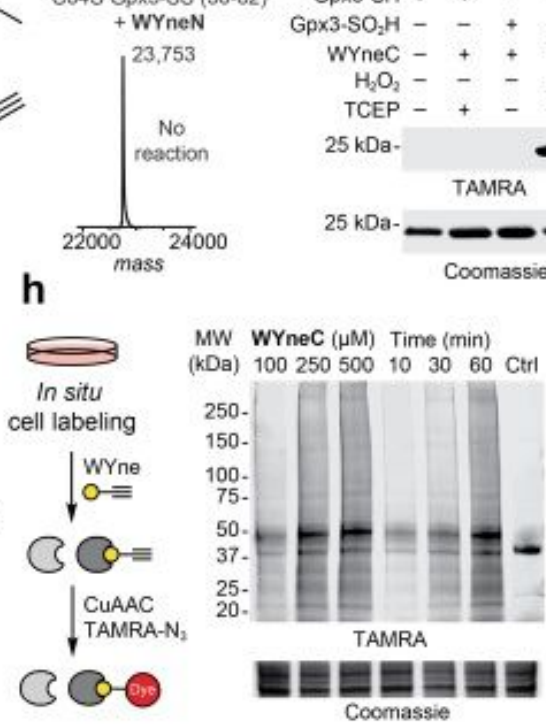

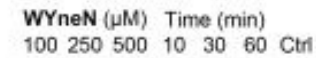

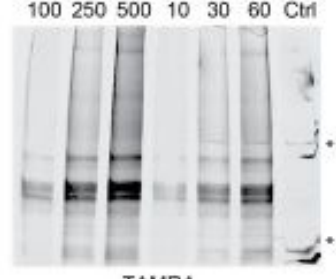

TAMRA

토토로르르를

Coomassie

Figure 2

WYne probe reactivity with sulfenic acid in complex biological settings. a, Physical property and kinetic profile of the WYne probes. b, Base-promoted hydrolysis facilitated cleavage of the TPP group from WYne probes. c, A small molecule sulfenic acid model (dipeptide-SOH) was used to kinetically evaluate WYne probes. $d$, Reaction of WYne probes and various sulfur species in $\mathrm{pH} 7.4$ aqueous-organic buffer. Owing to rapid kinetics, the reaction between WYneC and dipeptide-SOH (R-SOH) was performed at $\mathrm{pH} 4.9$ and the rate constant extrapolated to pH 7.4 (see Supplementary Methods). Reactivity with other biological sulfur species was not observed. e, Glutathione peroxidase 3 (Gpx3, from S. cerevisiae) as a model to study protein sulfenic acids. f, Intact protein MS analyses suggested that WYne probes exclusively 
targeted the sulfenic acid form of Gpx3 and not the thiol, sulfinic acid or disulfide forms. $\mathrm{g}$, In-gel fluorescence detection of Gpx3 sulfenic acid. h, In situ labeling of A549 cells with WYne probes showed time and dose-dependence.

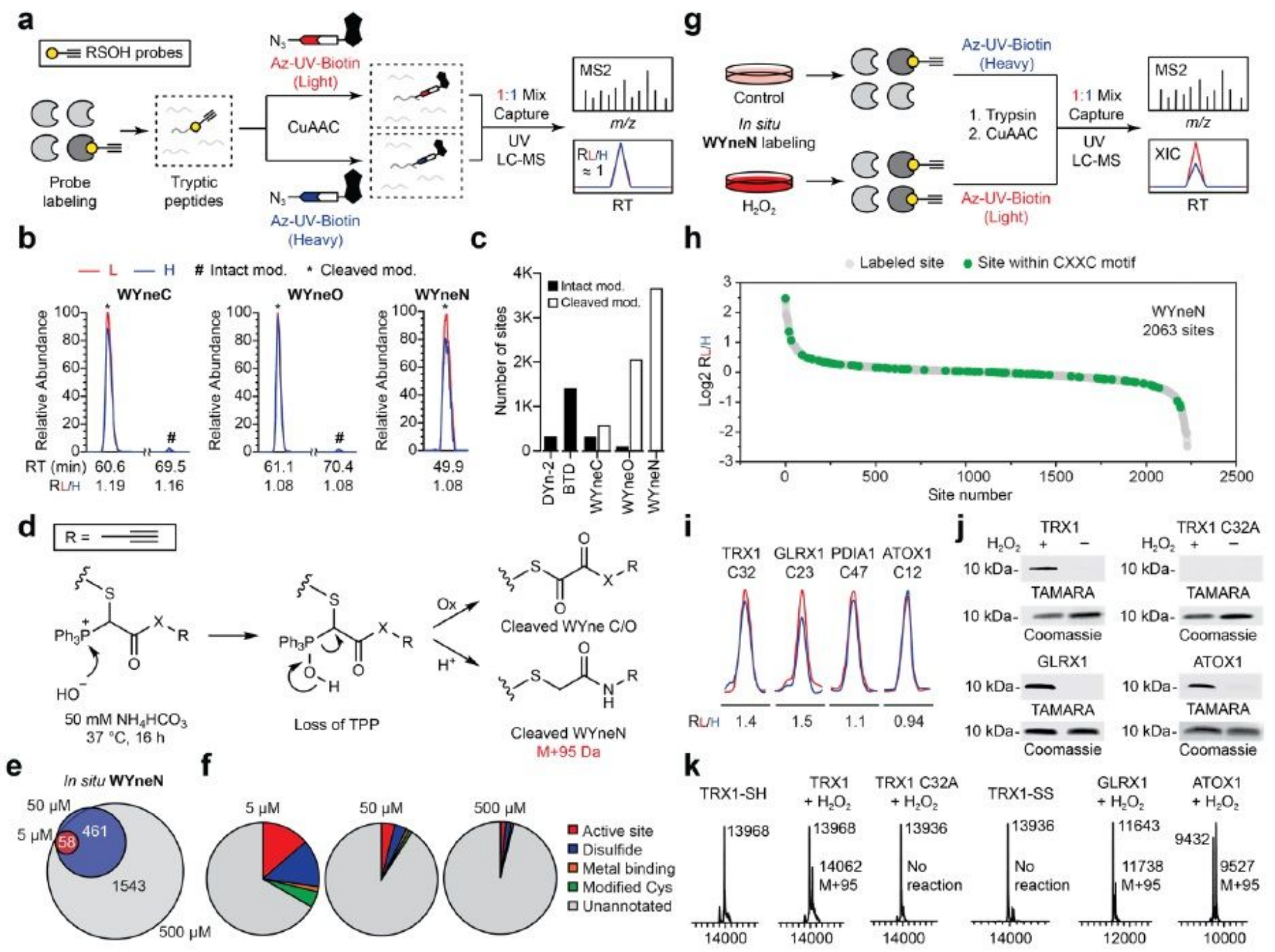

Figure 3

Profiling S-sulfenylation dynamics with WYneN in cells. a, Quantitative proteomics provides unequivocal identifications of SOH probe-labeling sites in the A549 proteome. b, Extracted ion chromatograms (XICs) of the isotopically labeled peptide (VPTANVSVVDLTCR derived from GAPDH) adducts derived from WYne probes. The intact (\#) and TPP-cleaved $\left({ }^{*}\right)$ adducts derived from each probe are shown in the same scale. c, Bar chart showing the number of sulfenylated sites profiled by using different $\mathrm{SOH}$-specific probes. $d$, Plausible mechanism for the formation of TPP-cleaved peptide adducts derived from WYne probes. e, Venn diagram showing the overlap of sulfenylated sites mapped by using WYneN with different doses for in situ labeling. $f$, Pie charts showing the percentage of functionally annotated cysteine sites from different WYneN-dosing groups. g, Quantitative chemoproteomic profiling of dynamic S-sulfenylation changes in A549 cells upon $\mathrm{H} 2 \mathrm{O} 2$ treatment. h, Rank order of the determined RL/H values of WYneNlabeled sites from A549 cells treated with or without $\mathrm{H} 202$. Those sites within redox-sensitive CXXC 
motifs were annotated in green color. i, Representative XICs showing for the changes in WYneN-labeled cysteines from TRX1, GLRX1, PDIA1 and ATOX1, with the profiles for light and heavy-labeled peptides in red and blue, respectively. j, Validation of CXXC protein labeling by in-gel fluorescence. $k$, Intact protein MS shows that TPP-cleavage occurs during the labeling of CXXC proteins.

a
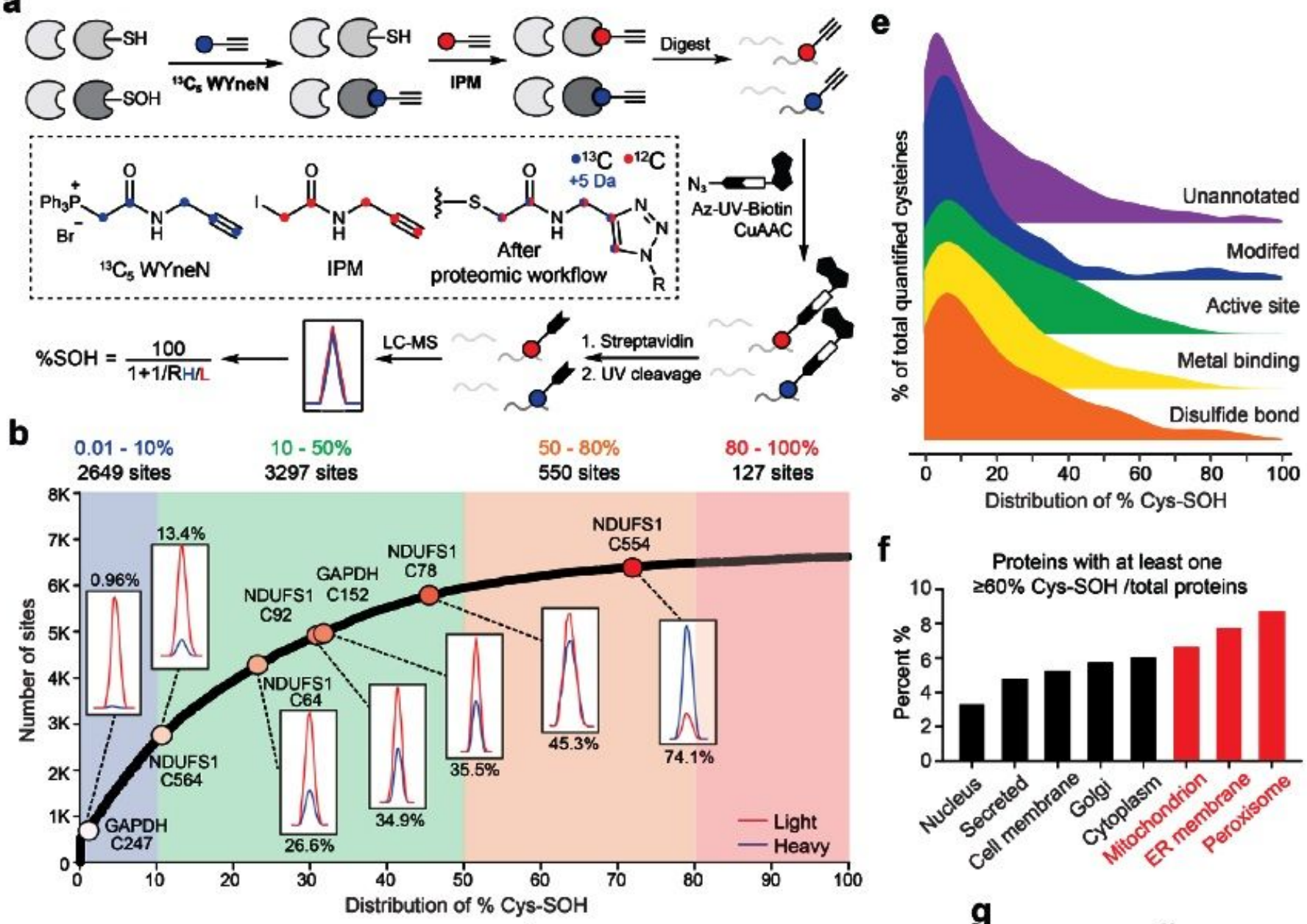

C $\% \mathrm{SOH}$

d $\% \mathrm{SOH}$
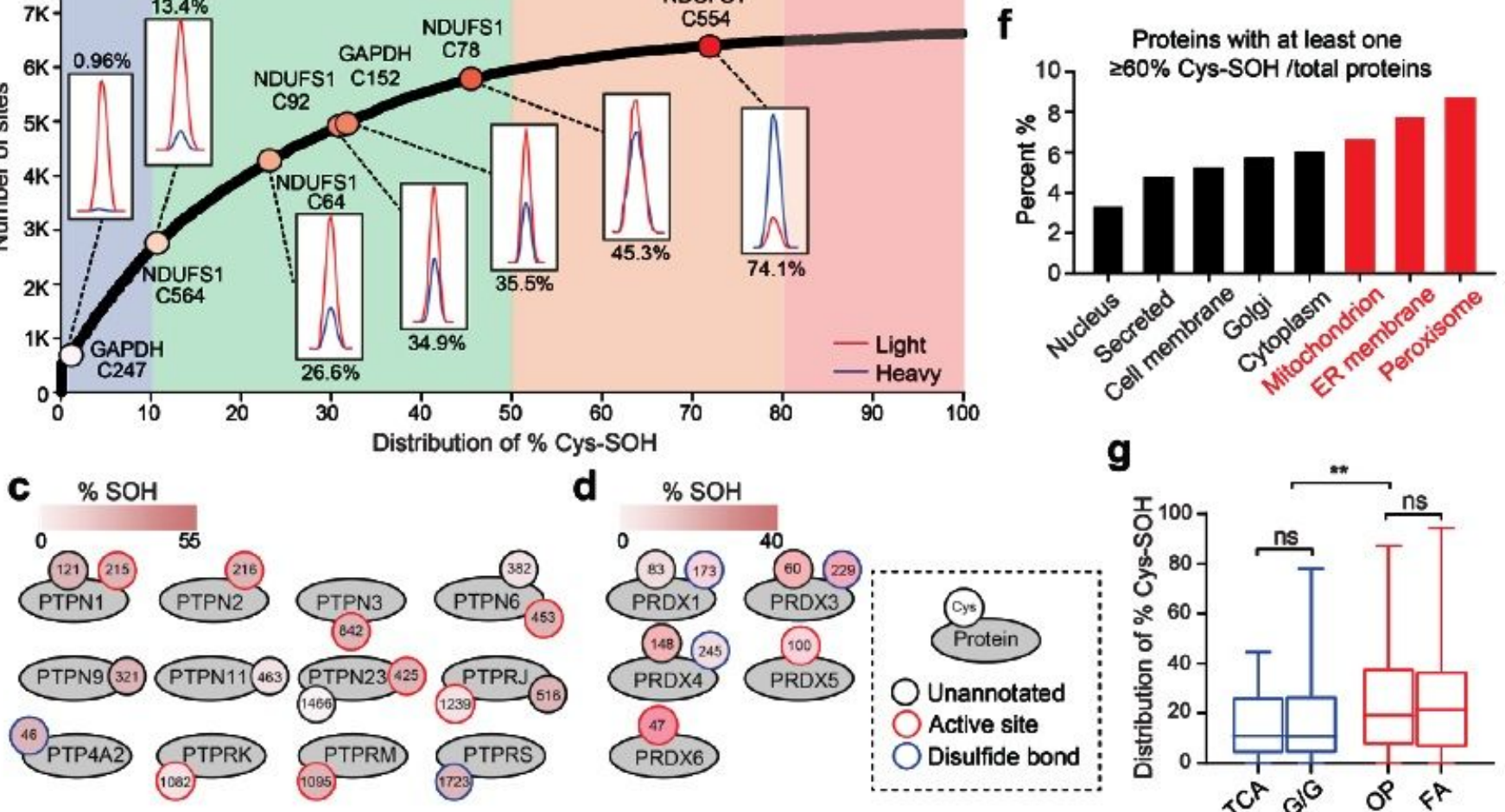

Figure 4

Proteome-wide analysis of cysteine sulfenic acid site stoichiometry. a, Quantitative determination of sitespecific occupancy of protein sulfenylation (\%SOH) in A549 cells. b, Distribution of the \%SOH values of 6,623 cysteines, which are classified into four groups as indicated. Insets: representative XICs showing for the relative levels of $\mathrm{SOH}$ (labeled with 13C5 WYneN) and SH (labeled with IPM) in cysteines from NDUFS1 and GAPDH. c-d, \%SOH of cysteines in protein tyrosine phosphatases (c) and peroxiredoxins (d). 
e, Density plots showing the distribution of \%SOH in cysteines with or without functional annotations. $\mathrm{f}$, Bar chart showing the distribution of proteins of high oxidation group (Site occupancy $₫ 60 \%$ ) according to subcellular locations retrieved UniProt. g, Box plot showing the \%SOH distribution of cysteines within proteins involved in selected gene ontology biological processes. TCA: tricarboxylic acid cycle, G/G:

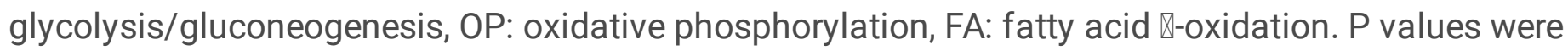
calculated using a two-tailed, unpaired t-test. ns, not significant, $* * P \leq 0.01$.

a

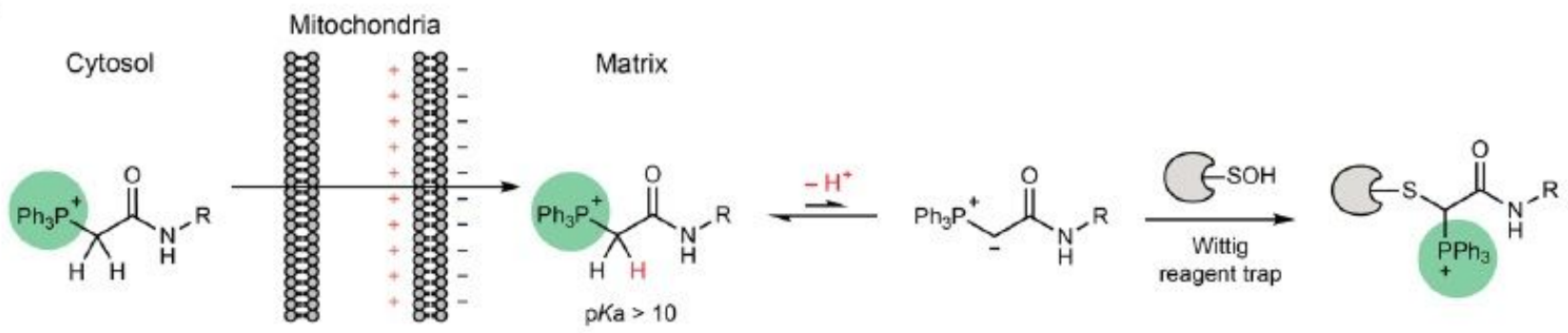

b

OMM IMM

C

BDP-WYneN10

MitoTracker

Merge $(R=0.85)$

Cationic and lipophilic
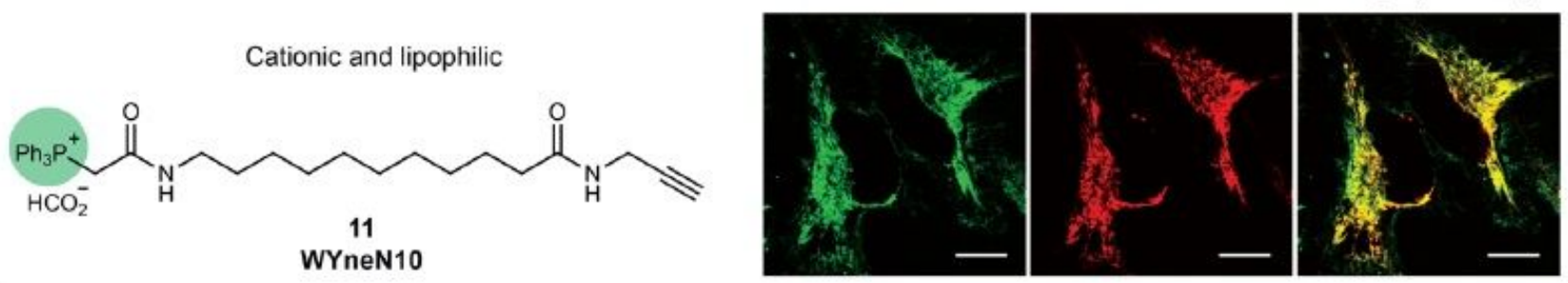

d

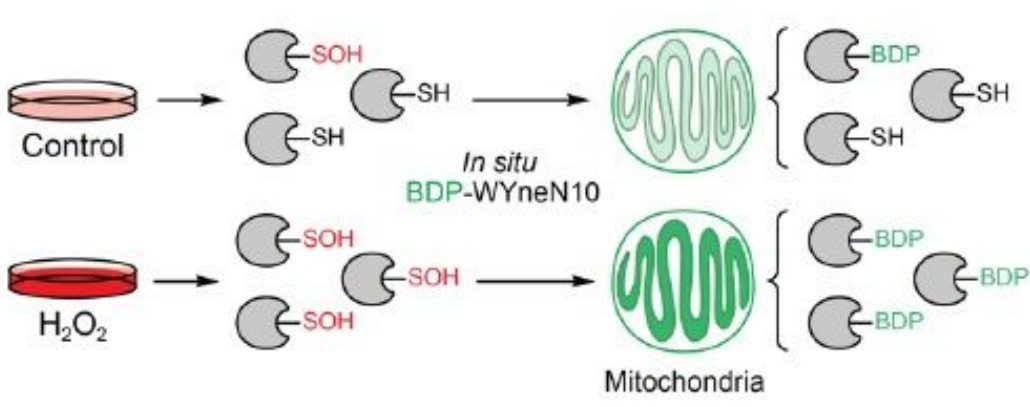

f
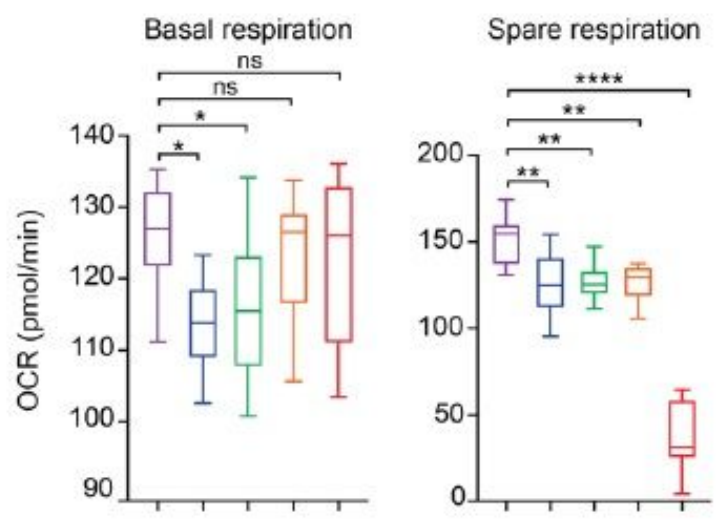

e
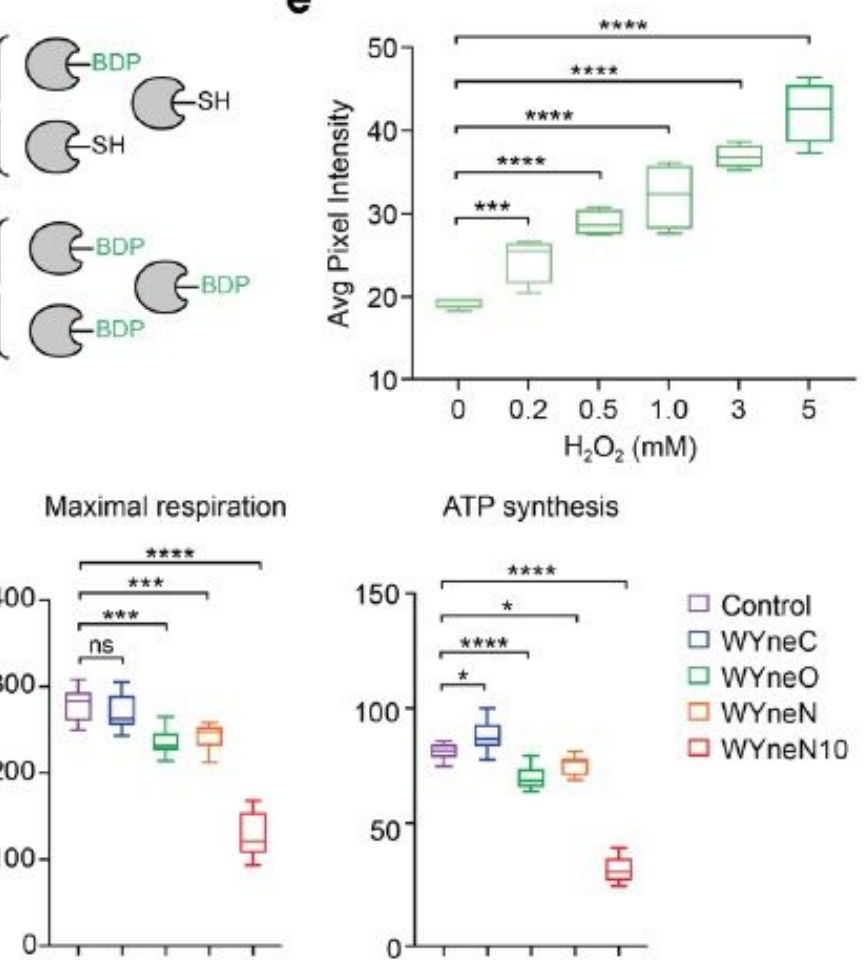

ATP synthesis

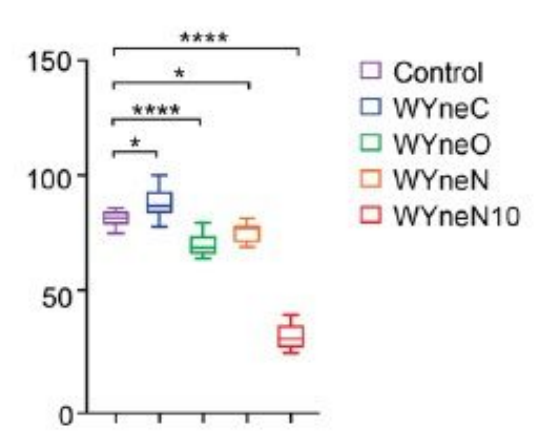

\section{Figure 5}

Imaging redox-dependent changes in mitochondrial cysteine oxidation. a, Amide derivative of Wittig reagents exists predominantly in protonated form, setting stage for enrichment and detecting S- 
sulfenylation in mitochondria. b, Structure of the mitochondrial targeting sulfenic acid probe WYneN10 with enhanced lipophilicity. c, Live cell imaging of BDP-WYneN10 suggested its mitochondrial localization in HeLa cells. MitoTracker: MitoTrackerTM Deep Red FM; R, Pearson's correlation coefficient. A scale bar of $20 \mu \mathrm{m}$ is shown. d, BDP-WYneN10 tagged S-sulfenylated proteins with fluorescence inside mitochondria. e, BDP-WYneN10 fluorescence responded to external oxidative stress $(0-5 \mathrm{mM} \mathrm{H} 2 \mathrm{O} 2)$ in live A549 cells $(n=4) . f$, WYneN10 disrupted mitochondrial respiration in A549 cells to a greater extent than other WYne probes. OCR, oxygen consumption rate. Error bars represent \pm s.e.m of biological replicates $(n=6)$. $P$ values were calculated using a two-tailed, unpaired t-test. ns, not significant, *P $\leq$

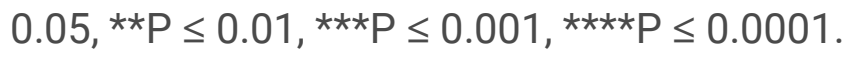


a

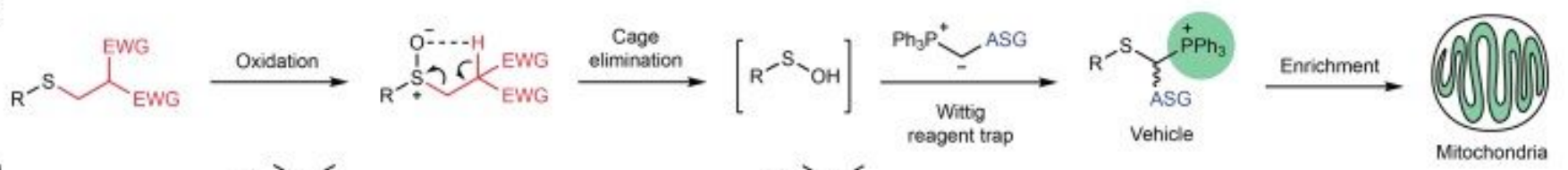

b

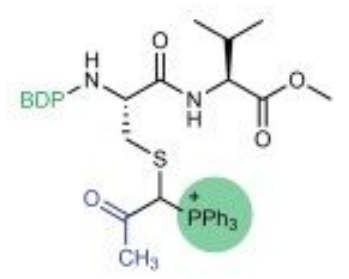

Vehicle 15

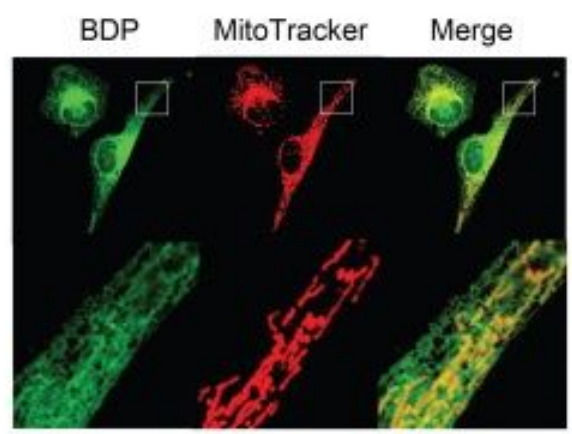

$R=0.55$

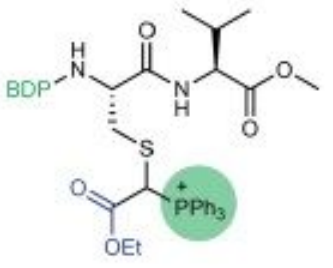

Vehicle 16

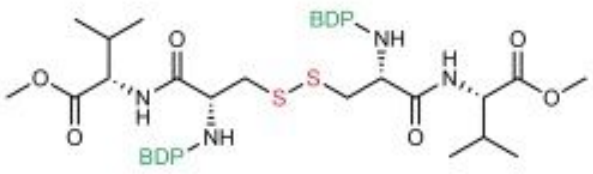

Disulfide 18

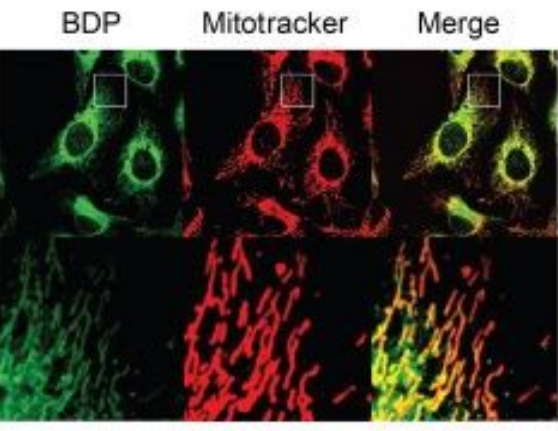

$\mathrm{R}=0.80$

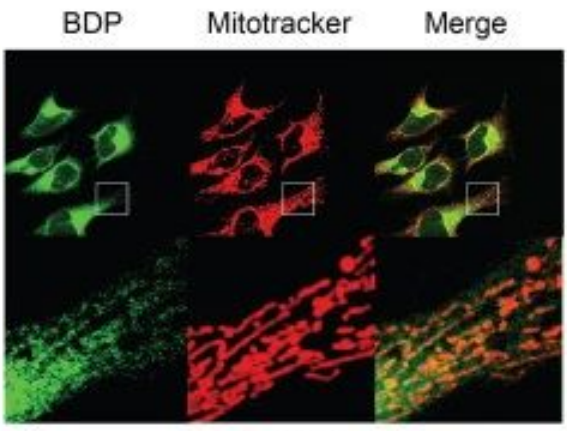

$R=0.39$

C

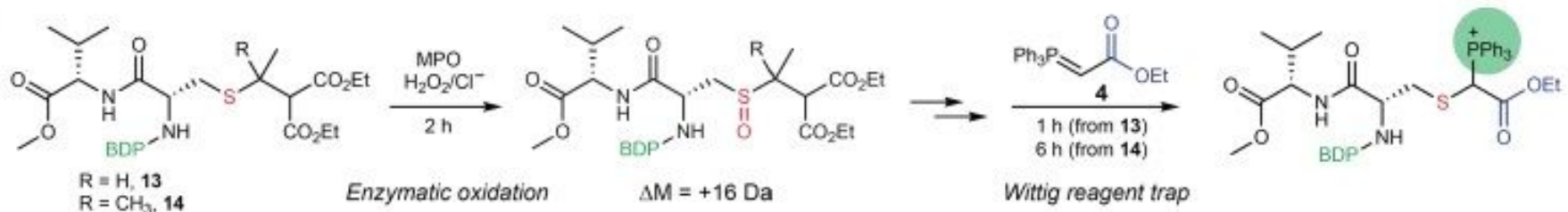

d<smiles>CCOC(=O)C([18O][Na])SCCCCCCCCCCC1=C(C)C(=O)C(OC)=C(OC)C1=O</smiles>
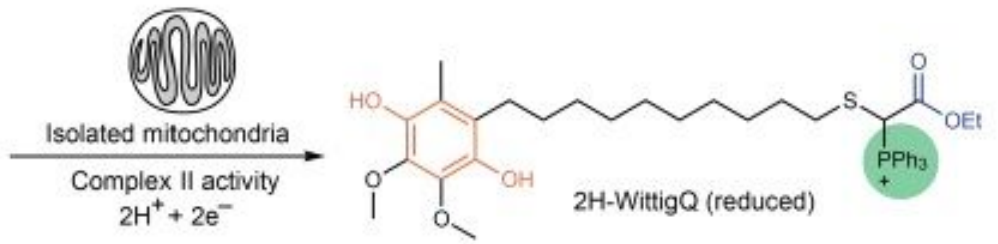<smiles>CCOC(OCC)C(C)SCCCCCCCCCCc1c(C)c(O)c(OC)c(OC)c1O</smiles>

e
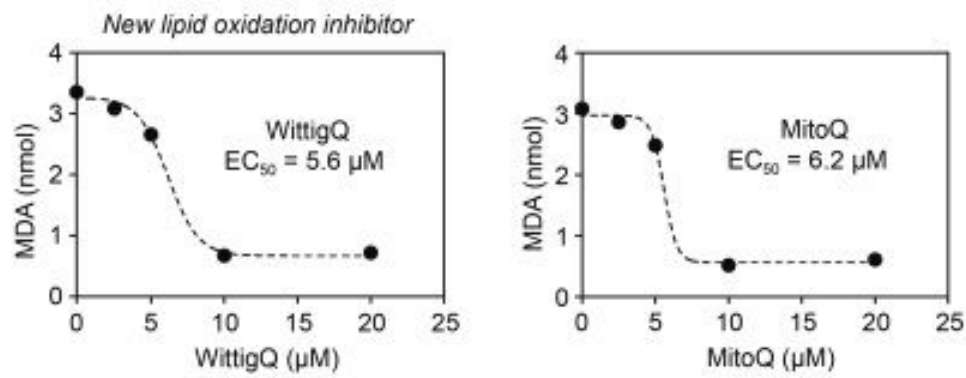

Figure 6

Redox-triggered in situ TPP generation for mitochondrial cargo delivery. a, General principle of a redoxcaged mitochondrial targeting system. b, Comparison of two different vehicle and a disulfide control compound in live cell imaging. BDP, BODIPY-tagged mitochondrial vehicles; MitoTracker: MitoTrackerTM Deep Red FM; R, Pearson's correlation coefficient calculated as the average of five regions of interest (ROIs) from representative cells. A zoom-in area of $20 \times 20 \mu \mathrm{m}$ was shown below each image. c, Enzymatic 
activation of the redox-caged sulfenic acids 13 and 14 by generation of $\mathrm{HOCl}$ from myeloperoxidase (MPO), followed by Wittig reagent trapping. d, Redox-caged sulfenic acid 19 was oxidized by $\mathrm{HOCl}$, reacted with Wittig reagent 4 to afford the mitochondria-targeting compound, WittigQ (20), which was actively reduced by complex II activity to the quinol form. e, WittigQ prevented lipid peroxidation in mitochondria isolated from HeLa cells at comparable efficacy to MitoQ. Representative data from two independent experiments are shown.

\section{Supplementary Files}

This is a list of supplementary files associated with this preprint. Click to download.

- ShietalSupplementary.pdf

- ShietalSupplementaryTable1.xlsx

- ShietalSupplementaryTable2.xlsx

- ShietalSupplementaryTable3.xlsx

- ShietalSupplementaryTable4.xIsx 